\title{
A trade-off between stress resistance and tolerance underlies the adaptive response to hydrogen peroxide
}

\author{
Basile Jacquel ${ }^{1234^{*}}$, Audrey Matifas ${ }^{1234}$, Gilles Charvin ${ }^{1234^{*}}$ \\ Affiliations: \\ 1) Department of Developmental Biology and Stem Cells, Institut de Génétique et de \\ Biologie Moléculaire et Cellulaire, Illkirch, France \\ 2) Centre National de la Recherche Scientifique, UMR7104, Illkirch, France \\ 3) Institut National de la Santé et de la Recherche Médicale, U964, Illkirch, France \\ 4) Université de Strasbourg, Illkirch, France \\ *: Corresponding authors: charvin@igbmc.fr, jacquelb@igbmc.fr
}

\begin{abstract}
$\underline{\text { Abstract }}$
In response to environmental stress, cellular defense strategies may be divided into two categories: those, as in homeostatic systems, that seek to maintain cell proliferation by degrading the stressor (i.e., resistance); and those that ensure cell survival (i.e. tolerance), even if this is often at the expense of cell proliferation. In this study, we have explored the genetic bases of the antagonism between resistance and tolerance during the response to hydrogen peroxide $\left(\mathrm{H}_{2} \mathrm{O} 2\right)$ in budding yeast. We show that inactivation of protein kinase $A$ (PKA) by $\mathrm{H} 2 \mathrm{O} 2$ signaling induces an abrupt transition from normal homeostatic function to a stress-tolerant state by protecting the growth machinery, hence maximizing cellular fitness in a changing environment. This model system paves the way for developing antiproliferative strategies that target both resistance and tolerance mechanisms to prevent relapse.
\end{abstract}

\section{$\underline{\text { Introduction }}$}


Cell responses to stress are based on multifaceted strategies that promote physiological adaptation in changing environments. A broad class of defense mechanisms against both environmental and endogenous insults -e.g. oxidative (Veal, Day, and Morgan 2007; Toledano et al. 2004; Toledano, Planson, and Delaunay-Moisan 2010), hyperosmolarity (Hohmann 2002)use sense-and-respond regulatory systems based to the homeostatic framework: cells attempt to restore a preexisting optimal cellular state by removing the cause of internal stress. In this scenario, the role of the homeostatic system is to maintain proliferation under stress, i.e. to reach a state of "stress resistance" (Brauner et al. 2016; Balaban et al. 2019). Whereas such regulatory mechanisms may drive excellent adaptive properties at steady-state (Goulev et al. 2017; Muzzey et al. 2009; Milias-Argeitis et al. 2011), they usually suffer from a number of limitations, including a limited homeostatic range and a slow response time -e.g. slow transcriptional activation (Goulev et al. 2017), which restricts the cell's ability to deal with abrupt unpredictable environmental changes.

An alternative defense scheme, referred to as "stress tolerance" (Brauner et al. 2016), has been described whereby a population of cells can survive a transient physiological threat without necessarily fighting the intrinsic cause of internal stress. In this framework, cell survival is usually associated with arrested proliferation and reduced metabolism, as commonly observed in the context of cellular dormancy (Gray et al. 2004), or in specific cases of heterogeneous cellular behaviors -e.g. bacterial persistence (Bigger 1944; Balaban et al. 2004), bet-hedging (Slatkin 1974; Levy, Ziv, and Siegal 2012). Stress response strategies can thus be split into those that seek to maintain cell proliferation under stress (resistance) versus those that ensure cell survival (tolerance), see Fig. 1A. The former may a priori maximize cellular fitness; however, their intrinsic limitations (e.g. homeostatic failure) expose the cells to damages and death. In the latter, cell survival comes at the expense of reduced cellular proliferation. Altogether, stress resistance and tolerance mechanisms thus appear as antagonistic cellular strategies of fitness maximization in response to stress, and, so far, how cells deal with such antagonism has not been investigated.

Redox homeostasis is an essential cellular feature that ensures reliable cellular function in cells experiencing redox perturbations of external and internal origin (Veal, Day, and Morgan 2007; Toledano et al. 2004). In yeast, the control of hydrogen peroxide concentration is achieved through a transcriptional sense-and-respond homeostatic system based on the Yap1 transcription factor (Lee et al. 1999; Delaunay, Isnard, and Toledano 2000; Kuge and Jones 1994). The Yap1 regulon drives the expression of about one hundred genes (Godon et al. 1998; Gasch et al. 2000; Lee et al. 1999), including antioxidant enzymes with somewhat overlapping $\mathrm{H}_{2} \mathrm{O}_{2}$ scavenging functions (Jiang and English 2006; Chae, Chung, and Rhee 1994; Pedrajas et al. 2000; Wong et al. 2002; Iraqui et al. 2009). Additional regulations participate in the restoration of internal redox balance: first, glycolysis rerouting to the pentose phosphate pathway (PPP) leads to increased production of NADPH (Ralser et al. 2007; Kuehne et al. 2015), which is the ultimate electron donor involved in $\mathrm{H} 2 \mathrm{O} 2$ buffering in the peroxidatic cycle (Hall, Karplus, and Poole 2009); then, the inhibition of the protein kinase A (PKA) pathway, which is a major hub for cell proliferation control (Broach 2012) and general stress response (Gasch et al. 2000), contributes to the adaptation to oxidative stress (Hasan et al. 2002) and is connected to the $\mathrm{H} 2 \mathrm{O} 2$ signaling response through various putative mechanisms (Boisnard et al. 2009; Molin et al. 2011; Bodvard et al. 2017; Roger et al. 2020). Therefore, the response to $\mathrm{H} 2 \mathrm{O} 2$ provides an ideal context in which to decipher whether and how resistance and tolerance mechanisms coordinate to shape an integrative adaptive stress response. 
To address this question, we have used live-cell imaging and microfluidics approaches to develop combined proliferation and survival assays that elicit the distinction between $\mathrm{H} 2 \mathrm{O} 2-$ resistant and tolerant cellular behaviors. Using a candidate-gene screen, we have classified the main players involved in $\mathrm{H} 2 \mathrm{O} 2$ stress response into functional categories that highlight their respective roles in adaptation to this stressor. Specifically, our study unraveled the existence of a strong antagonism between resistance and tolerance, which was exacerbated by mutations that affect NADPH fueling in the peroxidatic cycle (e.g., zwf1 and trr1 mutants). Further results allowed us to decipher the genetic bases of $\mathrm{H} 2 \mathrm{O} 2$ tolerance and its interplay with $\mathrm{H} 2 \mathrm{O} 2$ signaling. Altogether, our study thus revealed how the integrated cellular response to $\mathrm{H} 2 \mathrm{O} 2$ results from a trade-off between the homeostatic system that ensures cell proliferation and a mechanism that prevents cell death by protecting the growth machinery. This model system paves the way for developing anti-proliferative strategies in which both resistance and tolerance mechanisms could be independently targeted to improve therapeutic efficiency.

\section{$\underline{\text { Results }}$}

\section{Resistance and tolerance are distinct properties of the response to hydrogen peroxide}

To determine whether stress resistance and tolerance are relevant and distinct physiological properties of the $\mathrm{H} 2 \mathrm{O} 2$ stress response, we needed to establish whether yeast could tolerate (i.e., survive) $\mathrm{H} 2 \mathrm{O} 2$ doses beyond its ability to resist (i.e, to proliferate). To this end, we measured yeast resistance to $\mathrm{H} 2 \mathrm{O} 2$ as the threshold concentration beyond which cell proliferation arrests, which is also referred to as the Minimum Inhibitory Concentration (or MIC) (Brauner et al. 2016; Balaban et al. 2019). Importantly, since H2O2 is a relatively unstable compound, measuring the MIC for $\mathrm{H} 2 \mathrm{O} 2$ required a microfluidic chamber with continuous medium replenishment to maintain a constant concentration of the stressor previously described (Goulev et al. 2017).

Using time-lapse microscopy, we followed the proliferation of individual cells under continuous $\mathrm{H} 2 \mathrm{O} 2$ exposure (starting at $\mathrm{t}=0$ ). We found that, whereas cells could resume proliferation after a lag when exposed to $0.5 \mathrm{mM}$, a $1 \mathrm{mM}$ concentration entirely arrested cell growth, at least up to 16 hours (Fig. 1B). This result indicated that the MIC was between $0.5 \mathrm{mM}$ and $1 \mathrm{mM}$, in agreement with previous measurements(Goulev et al. 2017). In contrast, cell growth resumed after a $1 \mathrm{~h}$ stress pulse at $1 \mathrm{mM}$, indicating that a fraction of the cells managed to survive above the MIC, despite the lack of resistance (Fig. 1C). Interestingly, when varying the duration $\square$ of stress exposure (see Fig. 1D with $\square=4 \mathrm{~h}$ ), the survival fraction (see Material and Methods for details) displayed a similar decay at both $0.5 \mathrm{mM}$ and $1 \mathrm{mM}$ for $\square \leq 2 \mathrm{~h}$, suggesting that cell survival (i.e., tolerance) was somewhat independent of stress resistance (Fig. 1E). For $\square>3 \mathrm{~h}$, cell survival plateaued at $0.5 \mathrm{mM}$ but further decreased at $1 \mathrm{mM}$ due to growth recovery and population adaptation at $0.5 \mathrm{mM}$.

Altogether, the independent assessment of both proliferation and survival, as performed using our single-cell tracking methodology, revealed that resistance and tolerance are two distinct aspects of cell behavior in response to $\mathrm{H} 2 \mathrm{O} 2$.

\section{A genetic screen identifies mutants that combine a severe resistance defect with a hyper- tolerance phenotype}


To decipher the specific genetic determinants of tolerance and resistance, we designed a candidate-gene approach to systematically measure proliferation and survival in mutants that have been previously reported as "sensitive" to $\mathrm{H} 2 \mathrm{O} 2$. Notably, the survival assays at $0.5 \mathrm{mM}$ revealed that cells displayed a two-step response to stress (Fig. 1): an acute period, characterized by arrested growth and high mortality rate, followed by a steady-state, during which cell proliferation resumed, yet no further mortality was observed. Therefore, to further identify the genetic determinism associated with acute versus steady-state response, we compared survival and proliferation between stepping (i.e., acute stress and steady-state) and ramping (i.e., steady-state regime but no acute period, see Fig. 2A and Fig. S2A) stress patterns after a 4-hour exposure at 0.5mM (see Fig. 2A and Fig. S2B and (Goulev et al. 2017)). Cell proliferation was assayed by measuring the total biomass produced by surviving cells during stress exposure (Fig. 2B), and tolerance was calculated as a fraction of initial cells that resumed or maintained growth after stress exposure (Fig. 2C, see Materials and methods for details).

We screened 14 genes directly involved in $\mathrm{H} 2 \mathrm{O} 2$ defense such as Yap1, players associated with the peroxidatic cycle of 2-Cys peroxiredoxins (e.g. the peroxiredoxin Tsa1 (Wong et al. 2002), the thioredoxins Trx1/2 (Boisnard et al. 2009), the thioredoxin reductase Trr1 (Chae, Chung, and Rhee 1994)), other antioxidant genes (e.g the catalase Ctt1 (Guan et al. 2012) and the mitochondrial peroxidase Ccp1 (Jiang and English 2006)), genes driving the general Environmental Stress Response (or ESR, e.g. Msn2/4 (Boisnard et al. 2009; Hasan et al. 2002)), or specific mutants that were observed to be sensitive to $\mathrm{H} 202$ (such as the phosphodiesterase Pde2 (Hasan et al. 2002)) (see Fig. 2C and Fig. S2C). Results were displayed by plotting the mean survival and proliferation for each mutant analyzed under step and ramp conditions, Fig. 2D and $2 \mathrm{E}$.

We first verified that the yap1 $1 \Delta$ mutant displayed poor survival under these stress conditions (Fig. 2D and 2E), in agreement with previous studies (Delaunay, Isnard, and Toledano 2000). Then, we noticed that mutants could be classified into two different groups (see the shaded area on Fig. 2D and E): the first group (see the yellow shaded area on Fig. 2D and 2E) included mutants with mild (e.g., glr1 $\Delta$ ) to severe (e.g., ctt1 $\Delta$ ) proliferation and survival defects compared to WT. Importantly, in this group of mutants, both proliferation and survival increased significantly by comparing step to ramp assays (see the deviation off the diagonal in Fig. 2D and $2 \mathrm{E})$. This indicates that, despite their variable defects in the presence of acute stress, all mutants in this group displayed some level of adaptation at steady-state, hence were referred to as "adapters". In this category, proliferation and survival seemed to be affected somewhat proportionally, i.e., none of the corresponding genes had a role associated explicitly with either stress tolerance or resistance (with the probable exception of $p d e 2 \Delta$, which displayed a reduced tolerance yet a resistance similar or even superior to WT).

In contrast to the first category, we found a second group of mutants (referred to as "nonadapters") that spread along the diagonal on both survival and proliferation panels (Fig. 2D and $2 \mathrm{E}$ ). This indicated that submitting cells to a ramp did neither improve proliferation nor survival in these mutants, compared to a step, indicating that the corresponding genes were required to reach an adapted steady-state under stress. All these mutants also shared a greatly reduced resistance compared to WT and the group of adapters (Fig. 2E). Interestingly, genes in the nonadapter group were all associated with the biochemical pathway of NADPH-dependent 
peroxidases: following their reaction with $\mathrm{H} 2 \mathrm{O} 2$, oxidized Tsa1 and Tsa2 are reduced by the thioredoxins Trx1 and Trx2. Then, the thioredoxin reductase Trr1 catalyzes the reduction of Trx1 and Trx2 by NADPH, which is mainly synthesized upon glucose rerouting into the pentose phosphate pathway (PPP) by the glucose-6-phosphate dehydrogenase (G6PD, or Zwf1). Therefore, these results suggested that this biochemical pathway is the primary effector of $\mathrm{H} 2 \mathrm{O} 2$ homeostasis, unlike the catalase or the mitochondrial peroxidase.

To further test this hypothesis, we integrated a transcriptional Srx1pr-GFP-degron reporter in each mutant strain tested above (Srx1 encodes a sulfiredoxin, the expression of which is regulated by Yap1), as a proxy for the activation of Yap1 and as a marker of $\mathrm{H} 2 \mathrm{O} 2$ imbalance. Strains were submitted to a mild $0.1 \mathrm{mM} \mathrm{H} 2 \mathrm{O} 2$ step to ensure the cell's ability to drive a transcriptional response even in low-resistance mutants. Under these conditions, the WT strain displayed a transient burst of GFP fluorescence, followed by a recovery period leading to a steady-state level comparable to the pre-stress level (Fig. 2F). Such decay in fluorescence, which implies the deactivation of the Yap1 regulon, indicated that the internal $\mathrm{H} 2 \mathrm{O} 2$ balance was restored. By quantifying the amplitude of the fluorescence drop during the period from burst to steady-state, we defined an "H2O2 adaptation index" to quantitatively assess the ability of each mutant to restore $\mathrm{H} 2 \mathrm{O} 2$ balance (see sample data obtained with specific mutants on Fig. $2 \mathrm{~F})$. We plotted these measurements against the stress ramp resistance data displayed above (Fig. 2G). This analysis revealed that the restoration of $\mathrm{H} 2 \mathrm{O} 2$ balance was strongly impaired in the non-adapters defined above, in contrast to adapters, which were less severely affected. Furthermore, we found a good correlation between $\mathrm{H} 2 \mathrm{O} 2$ balance and resistance (Fig. 2G, $r^{2}=0.79$ ). Therefore, this analysis further demonstrated the specific role of the peroxiredoxin pathway in promoting both $\mathrm{H} 2 \mathrm{O} 2$ homeostasis and stress resistance at steady-state.

However, despite their belonging to the same group, we noticed that non-adapters mutants displayed very heterogeneous survival phenotypes, which could not be explained by differences in $\mathrm{H} 2 \mathrm{O} 2$ homeostasis (Fig. $2 \mathrm{H}$ and Fig. S2D). In addition, two of them (i.e., zwf1 $\Delta$, trr1 $\Delta$ ) had an unexpected $\sim 3-5$ fold higher survival fraction than that of the WT (Fig. 2D). Such dramatically increased tolerance was consistently observed during an $\mathrm{H} 2 \mathrm{O} 2$ exposure of up to $128 \mathrm{mM}$ (i.e., well above the MIC of the WT, Fig. S2E, and Fig. S2F), i.e., under conditions in which the cells were unlikely to trigger any active response. Therefore, our screen unraveled mutants in which a pronounced $\mathrm{H} 2 \mathrm{O} 2$ resistance defect, together with a loss of $\mathrm{H} 2 \mathrm{O} 2$ homeostasis, was associated with a hyper-tolerant phenotype, hence validating the necessity to disentangle mechanisms of resistance and tolerance during the $\mathrm{H} 2 \mathrm{O} 2$ stress response.

\section{A dynamic and reversible antagonism between redox imbalance, proliferation, and survival in the zwf1 $\Delta$ mutant}

How can mutants display both a severe resistance defect and a much stronger tolerance than WT? By focusing specifically on the zwf1 $\Delta$ mutant, we sought to address this conundrum by characterizing further the interplay between proliferation control, $\mathrm{H} 2 \mathrm{O} 2$ imbalance, and tolerance in this genetic background.

To this end, we quantified the $\mathrm{H} 2 \mathrm{O} 2$ imbalance using an Srx1pr-GFP-deg and the single-cell division frequency in cells exposed to growing mild concentrations of $\mathrm{H} 2 \mathrm{O} 2$ in WT and zwf1 cells (Fig. 3A-C and S3A). Unlike the WT, we observed a graded dose-dependent reduction in 
cell proliferation with increasing $\mathrm{H} 2 \mathrm{O} 2$ levels in the mutant, leading to a full division above $0.3 \mathrm{mM}$ (i.e., MICzwf1 $\Delta=0.3 \mathrm{mM}$ ). This growth defect under constant $\mathrm{H} 2 \mathrm{O} 2$ could not be explained by a putative limitation of NADPH since the trr1 $\triangle$ mutant, in which NADPH levels are not impaired, displayed the same phenotype as in the zwf1 $\Delta$ mutant (Fig S3B). Concomitantly, we observed a dose-dependent increase in the mean transcriptional activation of Srx1 under $\mathrm{H} 2 \mathrm{O} 2$ stress in the mutant, the magnitude of which was on average four times that of the WT (Fig. 3C). This observation was accompanied by a persistent nuclear localization of Yap1-GFP under $\mathrm{H} 2 \mathrm{O} 2$ stress, in contrast to the adaptive behavior observed in the WT, see Fig. S3C.

Interestingly, both cell division frequency and Srx1pr activation distributions obtained in the presence (i.e., $0.1 \mathrm{mM}$ ) or absence of $\mathrm{H} 2 \mathrm{O} 2$ stress displayed large cell-to-cell variability (Fig. 3D). Interestingly, we observed a negative correlation between the division frequency and Srx1 expression level in the mutant but not in the WT $\left(R^{2}(z w f 1 \Delta)=0.73\right.$ and $R^{2}(w t)=0.02$, respectively, at $0 \mathrm{mM} \mathrm{H} 2 \mathrm{O} 2$, see Fig. 3F). Hence, these results thus suggested the existence of an exacerbated antagonism between cell division and $\mathrm{H} 2 \mathrm{O} 2$ balance in the $z w f 1 \Delta$ mutant.

Cell proliferation arrests observed in the mutant could result either from an active growth control regulatory process or from cellular dysfunctions due to a potentially toxic $\mathrm{H} 2 \mathrm{O} 2$ imbalance. To test these hypotheses, we turned to fluorescent markers of protein oxidation to investigate whether proteins in the zwf1 $1 \Delta$ mutant were more oxidized than in WT and if this could compromise cellular function. To this end, first, we monitored the formation of fluorescence foci using the Tsa1-GFP fusion in cells exposed to a continuous $0.5 \mathrm{mM} \mathrm{H} 202$ concentration. Indeed, Tsa1 has been shown to induce the formation of supramolecular assemblies when super-oxidized in response to moderate to severe $\mathrm{H} 2 \mathrm{O} 2$ levels (Jang et al. 2004; Hanzén et al. 2016). In the WT, we observed a progressive formation of protein aggregates in response to stress, followed by a decrease that was presumably due to the adaptation to stress (Fig. 3E). Instead, in the zwf1 $\Delta$ mutant, aggregation was much faster and turned out to be irreversible over an 8-hour window. We obtained similar results with the Hsp104-GFP marker (Fig 3F), which was used as a generic marker of protein aggregation (Hsp104 is a disaggregase that binds misfolded aggregated proteins), and is known to form localized foci in response to $\mathrm{H} 2 \mathrm{O} 2$ (Erjavec et al. 2007). This suggested that part of the proteome is irreversibly oxidized in the zwf $1 \Delta$ mutant under stress. However, by releasing the cells that were exposed to $0.5 \mathrm{mM} \mathrm{H} 2 \mathrm{O} 2$ over 8 hours into a stress-free medium, we observed a progressive disaggregation of Hsp104-GFP that coincided with cell cycle re-entry(Fig. 3G and $\mathrm{H}$ ), indicating that the cellular oxidation was reversible and that the restoration of an internal $\mathrm{H} 2 \mathrm{O} 2$ balance elicited the recovery of cell proliferation. In further support to this, adding $5 \mathrm{mM}$ DDT to the medium upon $\mathrm{H} 2 \mathrm{O} 2$ stress release greatly accelerated both the disappearance of aggregates and cell cycle re-entry. Overall, these results suggested that, in the $z w f 1 \Delta$ mutant, cells' ability to proliferate was set by a regulatory mechanism dependent on the internal $\mathrm{H} 2 \mathrm{O} 2$ imbalance.

Similarly, we asked whether the level of $\mathrm{H} 2 \mathrm{O} 2$ imbalance could predict the tolerance to acute $\mathrm{H} 2 \mathrm{O} 2$ stress ( 1 hour at $64 \mathrm{mM}$ ) in $z w f 1 \Delta$ cells. Using the Srx1-GFP fusion as a read-out of initial internal $\mathrm{H} 2 \mathrm{O} 2$ imbalance at the time of exposure to stress (Fig. 3I, left panel), we plotted the survival fraction to the subsequent stress pulse as a function of the normalized Srx1-GFP expression level (data were pooled then binned in groups of $\mathrm{N}=46$ events). This experiment revealed that cells with a greater $\mathrm{H} 2 \mathrm{O} 2$ imbalance displayed enhanced tolerance, in agreement with the hyper-tolerant phenotype of the zwf1 $\Delta$ mutant compared to WT (Fig 3l). Altogether, these single-cell analyses further confirmed the strong antagonism between resistance, $\mathrm{H} 2 \mathrm{O} 2$ 
imbalance, and tolerance, and suggested the existence of a regulatory mechanism that couples all three features of the $\mathrm{H} 2 \mathrm{O} 2$ stress response.

\section{A thioredoxin-dependent PKA inhibition drives stress tolerance in part by protecting the growth machinery under $\mathrm{H} 2 \mathrm{O} 2$ stress}

The Ras - cyclic AMP (cAMP) - protein kinase A (PKA) pathway is a major proliferation control hub that transduces nutrient signals to regulate the transcriptional activation of ribosomes and protein synthesis (Broach 2012; Conrad et al. 2014; Tamaki 2007). In addition, PKA inhibition controls the stress response in part by inducing the nuclear relocalization of the Msn2/4 transcription factor (Boisnard et al. 2009; Jacquet et al. 2003), which drives the expression of hundreds of genes in response to various stressors (Gasch et al. 2000). In the specific context of oxidative stress, maintaining a high PKA activity has long been observed to induce $\mathrm{H} 2 \mathrm{O} 2$ sensitivity (Hasan et al. 2002), and recent studies proposed a mechanism whereby $\mathrm{H} 2 \mathrm{O} 2$ signaling through either peroxiredoxins or thioredoxins controls PKA activation and hence the downstream general stress response (Bodvard et al. 2017; Roger et al. 2020). Therefore, In the following, we sought to decipher the role of PKA in controlling both $\mathrm{H} 2 \mathrm{O} 2$ resistance and tolerance.

We reasoned that cell proliferation may be arrested in the zwf1 $\Delta$ mutant due to PKA inhibition. Hence alleviating this inhibition may rescue growth under stress conditions. To test this hypothesis, first, we deleted the phosphodiesterase Pde2, which drives the linearization of cAMP and thus inactivates PKA. By quantifying the nuclear shuttling of an Msn2-GFP fusion as a readout of PKA activation, we first checked that Msn2-GFP transiently relocalized to the nucleus in response to a $0.5 \mathrm{mM} \mathrm{H} 2 \mathrm{O} 2$ step in the WT (Fig. 4A), in agreement with the reversible proliferation arrest observed in Fig.1. We also verified that the nuclear localization was dependent on Pde2 (Fig. 4A and S4A). In contrast, in the zwf1 $\Delta$ mutant, Msn2-GFP was at least partially nuclear at all times during stress exposure, consistently with prolonged PKA inhibition (Fig. 4B). Strikingly, Msn2-GFP remained almost fully cytoplasmic throughout the experiment in the zwf1 $\Delta$ pde2 $\Delta$ double mutant (Fig. 4B), suggesting that deleting Pde2 in the zwf1 $\Delta$ mutant reactivated PKA.

Then, to directly check whether forced PKA activation could rescue cell growth, we compared the growth of these mutants and their $\mathrm{H} 2 \mathrm{O} 2$ imbalance (using an Srx1pr-GFP-deg reporter) in response to mild $\mathrm{H} 2 \mathrm{O} 2$ concentrations ranging from 0 to $0.3 \mathrm{mM}$, i.e, lower than the $\mathrm{MIC}$ of the zwf1 $\Delta$ mutant. We found that deleting Pde2 in a $z w f 1 \Delta$ context partially restored proliferation up to $0.2 \mathrm{mM} \mathrm{H} 2 \mathrm{O} 2$. However, it did not increase the MIC (Fig. $4 \mathrm{C}$ ), nor did it help restore the $\mathrm{H} 2 \mathrm{O} 2$ balance. A closer inspection of individual $z w f 1 \Delta$ pde $2 \Delta$ cells exposed to $0.3 \mathrm{mM} \mathrm{H} 2 \mathrm{O} 2$ revealed that part of the cell population (referred to as "non-responders" in the following) was unable to display any transcriptional activation of the Srx1pr during stress, unlike the zwf1 $\Delta$ mutant, in which all cells responded (Fig. 4D). In addition, only the non-responders were unable to resume proliferation after stress removal. Altogether, these results suggested that the increased resistance conferred by the Pde2 deletion in the $z w f 1 \Delta$ pde2 $\Delta$ double mutant induced a substantial decrease in cell tolerance.

To test this hypothesis directly, we made a step-stress cell survival assay and we found that the pde2 $\Delta$ deletion was epistatic to $z w f 1 \Delta$ for survival at $0.5 \mathrm{mM} \mathrm{H} 2 \mathrm{O} 2$ step (Fig. $4 \mathrm{E}$ ), as well as at much higher stress concentrations (Fig. S4C). Additional experiments using ramping stress exacerbated the genetic interactions between pde2 $\Delta$ and $z w f 1 \Delta$ (since the pde2 $\Delta$ mutant has 
no tolerance defect in ramps), especially when cAMP was added to the medium (Fig 4F). Altogether, these results demonstrated that the $\mathrm{H} 2 \mathrm{O} 2$ hyper-tolerance observed in the $z w f 1 \Delta$ mutant required PKA inhibition.

How is the internal $\mathrm{H} 2 \mathrm{O} 2$ signal relayed to mediate PKA inhibition in response to stress? Recent studies have proposed that peroxiredoxins in their oxidized form (or, alternatively, thioredoxins) may inactivate PKA through a redox-dependent mechanism (Bodvard et al. 2017; Roger et al. 2020). To test this model in the context of stress tolerance, we investigated whether the deletion of thioredoxins or peroxiredoxins would be epistatic to the zwf1 $\Delta$ mutant in survival assays. When submitted to a step stress, we found that the hyper-tolerant phenotype of both $z w f 1 \Delta$ and $\operatorname{trr} 1 \Delta$ mutants under step stress was abolished in the $\operatorname{tr} x 1 / 2 \Delta z w f 1 \Delta$ and $\operatorname{tr} x 1 / 2 \Delta \operatorname{trr} 1 \Delta$ mutants, respectively, and similar results were obtained with ramps (Fig. 4G). Importantly, the quantification of nuclear Msn2-GFP localization confirmed that PKA was no longer inhibited in the trx1/2 $z$ wf1 $\Delta$ mutant under stress (Fig.S4D). Furthermore, knocking out all three peroxiredoxins Tsa1, Tsa2, and Ahp1, but not Tsa1 alone, abolished the hyper-tolerant phenotype of the zwf1 $\Delta$ mutant (Fig. S4E). Deleting Tsa1 alone also failed to prevent PKA inhibition in the zwf1 $\Delta$ background (Fig.S4D), suggesting that Tsa1 itself is not the relay mediating PKA inactivation. In this scenario, the loss of hyper-tolerance observed in the tsa1 $\Delta$ tsa2 $\Delta$ ahp1 $\Delta$ zwf1 $\Delta$ mutant could be interpreted as the fact that thioredoxins may no longer be oxidized in the absence of their major substrate (i.e. peroxiredoxin). Therefore, these results suggested that thioredoxins are the main player relaying $\mathrm{H} 2 \mathrm{O} 2$ signals to PKA and eliciting stress tolerance.

Last, we wondered by which mechanism PKA inhibition would drive cellular protective effects leading to stress tolerance. To this end, we sought to identify genes downstream of PKA whose deletion would abolish the hyper-tolerant phenotype of the zwf1 $\Delta$ mutant (Fig. $4 \mathrm{H}$ ). We found that an msn2/4 $\Delta$ mutant was epistatic to the zwf1 $\Delta$ mutant for tolerance (Fig. 4l), presumably because of the pleiotropic roles of Msn2/4 in the general stress response (Gasch et al. 2000). Interestingly, tolerance was also abolished in the triple mutant pat1 $\Delta$ dhh1 $\Delta$ zwf1 $\Delta$ (Fig. 4I). This suggested that Pat1 and Dhh1, which are involved in the formation of P-bodies and stress granules (Nissan et al. 2010; Decker and Parker 2012), may protect the translation machinery in the presence of $\mathrm{H} 2 \mathrm{O} 2$ - even though no such epistasis was observed by mutating Gcn2, which controls translation initiation (Garcia-Barrio et al. 2000), see Fig. 4H). To check this hypothesis further, using a Pat1-GFP fusion as a reporter of P-bodies formation, we observed a stronger aggregation of this marker in the zwf1 $1 \Delta$ mutant compared to WT under stress, consistently with its highly tolerant phenotype (Fig. $4 \mathrm{~J}$ and $\mathrm{K}$ ). Conversely, any further mutation that prevented PKA inhibition decreased the aggregation level of Pat1-GFP back to the WT level or lower (Fig. $4 \mathrm{~J}$ and $\mathrm{K}$ ). This result suggested that P-bodies and stress granules formation contributes to the establishment of tolerance by protecting the growth machinery under $\mathrm{H} 2 \mathrm{O} 2$ stress.

Altogether, the analysis of the genetic determinism of the hyper-tolerant phenotype in the $z w f 1 \Delta$ mutant thus unraveled the antagonistic roles of PKA in orchestrating the response to $\mathrm{H} 2 \mathrm{O} 2$ stress: whereas the growth inhibition mediated by PKA leads to cell survival in part by protecting the translation machinery, it comes at the expense of the $\mathrm{H} 2 \mathrm{O} 2$ homeostatic system, which requires cell growth to function (Fig. 5).

\section{Discussion}


In this study, we have used a single-cell time-lapse methodology to investigate the mechanisms that underlie the adaptation to oxidative stress in budding yeast. Our analysis reveals that the cells' ability to grow under stress (resistance) is distinct from their ability to survive the stressor (tolerance). We show that each of these aspects is determined by different mechanisms. Importantly, this distinction could not be operated without the use of longitudinal analyses, which are essential to track the fate of individual cells in response to stress. In contrast, $\mathrm{H} 2 \mathrm{O} 2$ "sensitivity" assays based on plate measurements, even though they can be very quantitative, do not allow one to discriminate between resistance and tolerance since both control cellular fitness and biomass production. Hence classical analyses could not correctly assess the behavior of mutants, such as the hyper tolerant $z w f 1 \Delta$, that were previously characterized as "sensitive" to $\mathrm{H} 2 \mathrm{O} 2$.

The lack of distinction between tolerance and resistance might in part explain why the detailed role of classical redox players in the adaptation to oxidative stress has remained elusive, despite an extensive characterization of their specific biochemical functions. Here our candidate-gene genetic screen allows us to classify mutants according to their respective functional roles. Importantly, it reveals that specific mutations may impair the $\mathrm{H} 2 \mathrm{O} 2$ homeostatic core, hence decreasing cellular resistance, yet contributing to enhancing cellular tolerance to $\mathrm{H} 2 \mathrm{O} 2$. Unraveling this antagonistic role is critical to reaching an integrative understanding of the $\mathrm{H} 2 \mathrm{O} 2$ defense mechanisms.

Our study clearly unravels the existence of distinct stress response mechanisms that drive different cellular behaviors: resistance, which marks the ability to grow under stress, appears to be directly linked to the ability to restore an internal $\mathrm{H} 2 \mathrm{O} 2$ balance; tolerance, which can be defined as the ability to survive under stress, is a separate property that requires PKA inhibition. Due to the intrinsic limitations of the $\mathrm{H} 2 \mathrm{O} 2$ homeostatic system, cellular resistance is maximized under either low $\mathrm{H} 2 \mathrm{O} 2$ levels or when $\mathrm{H} 2 \mathrm{O} 2$ levels are progressively increased (i.e. ramping stress), see Figure 5. In contrast, in response to acute $\mathrm{H} 2 \mathrm{O} 2$ steps larger than $0.6 \mathrm{mM}$, tolerance is the primary defense mechanism. The coordination of these two processes is what drives the overall response to $\mathrm{H} 202$. Tolerance and resistance display some functional overlap at intermediate stress levels. Indeed, the transient activation of the Yap1 regulon (which is a readout of the homeostatic system activation) is concomitant with the inhibition of cell growth, as monitored using cell cycle arrest and the activation of Msn2-GFP. Yet, these two mechanisms are antagonistic since resistance aims at maximizing cellular fitness by limiting cellular damages and therefore ensuring cell growth, while tolerance is based on the downregulation of cell growth. Thus, this study provides a unique example of a stress response strategy based on a tradeoff between growth and survival.

In response to oxidative stress, the role of PKA has long been reported and was recently refined by providing genetic and biochemical evidence that its inhibition is mediated either by peroxiredoxins or thioredoxins, which relay H2O2 signals (Bodvard et al. 2017; Roger et al. 2020). The model generally assumed in the literature is that PKA inhibition serves to trigger the environmental stress response through the activation of Msn2/4. Here, we propose that it is required to protect the growth machinery by forming $\mathrm{P}$-bodies and stress granules, which would be otherwise exposed to $\mathrm{H} 2 \mathrm{O} 2$ and hence generate damages that would compromise cell survival. 
The diverse functions of peroxiredoxins (and related partners involved in this biochemical pathway) have been thoroughly documented. Among them, the role of Tsa1 as an $\mathrm{H} 2 \mathrm{O} 2$ signaling molecule (in particular for PKA inhibition) has received considerable attention. This contributed to toning down its importance as an $\mathrm{H} 2 \mathrm{O} 2$ scavenging enzyme. Here, our results emphasize that both functions are equally important: the peroxidase function is essential (in contrast to the catalase $\mathrm{Ctt1}$ ) for homeostatic system function and hence resistance, whereas Tsa1/Trx signaling to PKA is the key mechanism that drives tolerance, see Figure 5.

Interestingly, while stress response mechanisms are often associated with tolerance in antibiotics research, studies that focus on physiological homeostatic systems instead try to understand the determinants of resistance. Our analysis of the $\mathrm{H} 2 \mathrm{O} 2$ stress response shows that resistance and tolerance are intertwined elements with overlapping molecular bases, both of which contribute to cellular fitness. Most importantly, mutations that impair resistance (e.g., zwf1 $\Delta$ ) do not necessarily affect tolerance and may even enhance it. Conversely, targeting tolerance by preventing PKA inhibition (e.g., pde2) does not necessarily impair cellular resistance (pde2 $\Delta$ mutant has no growth defect in $\mathrm{H} 2 \mathrm{O} 2$ ramps). However, altering both resistance and tolerance leads to a strong fitness defect no matter the temporal stress profile. Many cancer therapeutic strategies attempt to impair cellular functions that lead to cellular resistance defects. Yet, tolerance mechanisms can be responsible for relapses after the treatment. Targeting both aspects of stress response could provide an avenue to prevent relapse in cancer treatments.

\section{Material \& Methods}

\section{Strains and plasmids}

All strains were issued from the S288C background (Sikorski and Hieter, 1989; Huh et al., 2003), derived from BY4741 or BY4742. The list of strains is detailed in Supplementary file 1. Simple mutant strains were all issued from the BY4742 delta collection (invitrogen). The trx $1 \Delta$ trx2 2 and the tsa1 1 tsa2 $\Delta a h p 1 \Delta$ strains were gifts from the Toledano Lab and were also derived from S288c. The strain msn2 $4 m s n 4 \Delta$ was Dr. Li Wei and was also derived from S288c. The strain pat $1 \Delta d h h 1 \Delta$ was a gift from the Roy Parker lab and was derived from S288c. All strains have been checked by PCR with oligos targeting the kanMX4 cassette.

The transcriptional reporter strains carrying the SRX1pr-sfGFP-deg were generated by a onestep cloning-free method (Huber et al., 2014) in the corresponding mutant strain issued from the BY4742 delta collection (invitrogen).

The double mutants $(X \triangle Z W f 1 \Delta::$ natMX4) were obtained by substituting the entire ZWF1 gene by a natMX4 cassette in the corresponding $X \Delta:: k a n M X 4$ strain from the BY4742 delta collection

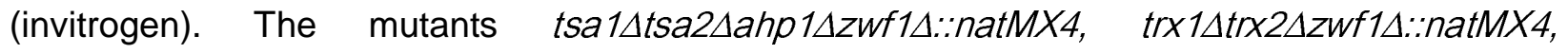




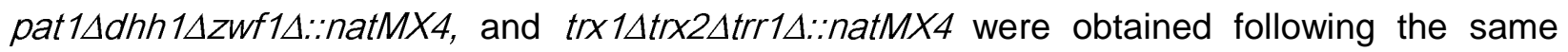
procedure, substituting the ZWF1 or the TRR1 gene by a natMX4 cassette in the corresponding strain. The triple mutant msn2 $\Delta m s n 4 \Delta z W f 1 \Delta$ was obtained by crossing X-03 (msn2 $\Delta m s n 4 \Delta$ ) with BJ2-28 (zwf1 $)$.

The protein fusion GFP strains were obtained from the BY4741 Invitrogen collection. The strains HSP104-GFP $z W f 14::$ natMX4 was obtained by substituting the entire ZWF1 gene by a natMX4 cassette in the corresponding protein fusion GFP strain (from invitrogen).

The PAT1-GFP::URA, PAT1-GFP::URA zwf1 , PAT1-GFP::URA zwf1 GFP::URA pde2 $\Delta$ and PAT1-GFP::URA tr $x 1 \Delta t r \times 2 \Delta$ strains have been obtained by fusing GFP at the $\mathrm{C}$ terminal of the PAT1 gene in the corresponding mutant strains.

\section{Microscopy}

Microfabrication and microfluidics setup

The microfluidic chips design was the same as the one used and described in Goulev et al., eLife, 2017. Microfluidics chips were PDMS replicas of the master mold. The chip was covalently bonded to a $24 \times 50 \mathrm{~mm}$ coverslip with the use of a Plasma activator (Diener, Germany). The microfluidic chip was connected to a peristaltic pump (Ismatec, Switzerland) within one hour following the plasma activation with Teflon tubing. The rate of the pump was set between 20 and $60 \mu \mathrm{L} / \mathrm{min}$. Switches of media were done manually. Linear ramps of medium containing $\mathrm{H}_{2} \mathrm{O}_{2}$ were done as described in Goulev et al., Methods Cell Biol 2018.

Growth medium and $\mathrm{H} 2 \mathrm{O} 2$ preparation

For bulk cultures and microfluidics experiments, yeast was grown at $30^{\circ} \mathrm{C}$ in synthetic complete $2 \%$ dextrose (SCD from ???) supplemented with amino-acids.

The $\mathrm{H}_{2} \mathrm{O}_{2}$ (Hydrogen peroxide solution 35wt. \% in $\mathrm{H}_{2} \mathrm{O}$, 349887-500 ML, Sigma) solutions were prepared as described in Goulev et al., eLife 2017. Briefly, solutions were prepared no more than $1 \mathrm{~h}$ before the experiment. $\mathrm{H}_{2} \mathrm{O}_{2}$ was mixed with SCD media at the required concentration and kept on ice prior to the experiment. The $\mathrm{H}_{2} \mathrm{O}_{2}$ concentration was checked at the end of the experiment and we found no

Time-lapse microscopy

For time-lapse imaging, cells were pre-grown overnight at $30^{\circ} \mathrm{C}$. Overnight cultures were then diluted and grown for 4 to $6 \mathrm{~h}$ to mid-log phase and were injected in the microfluidics device. Cells were let at least $1-2 \mathrm{~h}$ before the beginning of the experiment. Images were taken using an inverted Zeiss Axio Observer Z1 or a Zeiss Axiovert. The focus was maintained using a software custom algorithm developed on MatLab. Fluorescence images were taken using a LED light (CoolLed, LumenCor). The objectives used were a 63x N.A. 1.4 objective (with Zeiss Axio Observer Z1) or a 40x N.A. 1.4 objective (Zeiss Axiovert) and an EM-CCD Luca-R camera 
(Andor). Multi-Position imaging was enabled by an automated stage (up to 80 positions). GFP was excited at $\mathrm{XX} \mathrm{nm}$ after passing through an excitation filter () and fluorescence was then collected using an emission filter (). For all experiments, one image was acquired every 10 or 15 min except for the determination of the SRX1pr-GFP-deg expression. For that, one image was taken every 5 or $10 \mathrm{~min}$.

The temperature was set to $30^{\circ} \mathrm{C}$ during the whole experiment using both a custom objective heater (controlled with a 5C7-195, Oven Industries) and a holder heater (controlled using a custom Arduino module).

\section{Cell segmentation and cell mapping}

Raw images were imported and processed on MatLab using a free access custom software (phyloCell, available on GitHub, Charvin 2017). The software was used to segment and track cells over time. Segmentation and tracking were corrected manually with the phyloCell interface when needed. Data analysis was then done following the detailed procedures developed below.

\section{Data analysis}

All the data analyzed in this study came from at least two independent experimental replicates. All the data have been extracted from microfluidics experiments done in a controlled and stable environment (see below).

Analysis of physiological parameters

\section{Post-stress survival fraction}

For post-stress survival quantifications, only the cells present at the beginning of the stress were included. All cells born during the stress or after the stress were excluded. Following this procedure, the post-stress survival fraction was assessed independently of the proliferative capacity of cells under stress. The survival fraction was thus assessed by determining the fraction of cells born before stress exposure able to form a proliferate after the release of stress. To evaluate their ability to reproliferate after stress, we checked whether each cell was forming two new buds within the $15 \mathrm{~h}$ following the stress release. Counting two buds enable to not include cells that remain stuck in G2/M during their first post-stress division due to a DNAdamage checkpoint arrest (Goulev et al., 2017).

Each single survival fraction included at least 100 cells (except for trx $1 / 2$ delta where some conditions included only between 50 to 100 cells). Importantly, we are conscious that very small fractions of cells may not be captured in our microfluidic assay due to the relatively small number of cells $\left(\sim 10^{\wedge} 2\right.$ to $\left.10^{\wedge} 3\right)$. Survival fraction of persister cells as small as $1 / 10^{\wedge} 5$ cells have been reported in different contexts.

\section{Normalized proliferation during stress}

Proliferation under stress was assessed by measuring the fold cell number during the stress period for each cell present at the beginning of the stress period, based on the segmentation and the mapping of single-cells. Importantly, cells were included only if they had survived after the stress to avoid the possibility that the proliferative measurement would reflect a survival 
deficiency rather than a proliferative defect. Following this procedure, the proliferation under stress was assessed independently of the survival fraction. The readout was called 'Normalized cell proliferation' since it reflected a 'fold increase'.

\section{Proliferative capacity}

The proliferative capacity represents the normalized proliferation during stress relative to the WT score in the same stress conditions.

\section{Bud to bud frequency quantification}

The bud to bud frequency was calculated as the inverse of the time measure between the formation of two successive buds (proxy of cell cycle duration).

\section{Colony proliferation}

Colony proliferation was measured by counting the number of cells over time under stress normalized by the number of cells present at the onset of stress exposure.

\section{MDK99}

The minimal duration for killing 99\% of the population has been assessed based on 2 to 4 independent microfluidic experiments for each condition tested. The survival fraction was assessed as described in the section 'Post-stress survival fraction'. The represented MDK99 corresponds to the mean survival fraction of the different replicates. Each independent replicate included at least 100 cells. Importantly, the significance of the difference between the survival fraction measured and the fixed survival limit of $1 \%$ has not been tested statistically due to the low number of replicates (2 for some conditions). The observed MDK99 must therefore be viewed as a qualitative assay. We explain this choice by a large number of experiments that would have been required to screen the whole range of $\mathrm{H} 2 \mathrm{O} 2$ concentrations and durations with at least 3 replicates. However, we found that the MDK99 measured for the zwf1 delta strain was at least 4 times higher than the one required to kill the WT population from 1 to $64 \mathrm{mM}$. Such differences over several log2 scales strongly suggest the sufficiency of our qualitative assessment.

\section{MIC}

The minimal duration to inhibit the growth of the population was routinely assessed by the incapacity of cells to recover an exponential growth within $12 \mathrm{~h}$ following stress exposure. We tested in three independent replicates that at $0.3 \mathrm{mM}$ (the smallest dose at which a zwf1 delta population did not grow) that the growth did not recover within $24 \mathrm{~h}$ following stress exposures under $\mathrm{H} 2 \mathrm{O} 2$. A $12 \mathrm{~h}$ arrest was therefore considered as a 'definitive' arrest in the case the stress would be maintained.

Fluorescence analysis

\section{Yap1 and Msn2 nuclear localization quantification}

Camera background was first subtracted from the GFP signal. Yap1-sfGFP and Msn2-GFP nuclear localization were then quantified as described in Cai et al., Nature, 2008. Briefly, the nuclear localization score was measured by the difference between the mean intensity of the 5 
brightest pixels in the segmented cell and the mean intensity of all other pixels in the same segmented area.

\section{Quantification Hsp104-GFP, Tsa1-GFP, and Pat1-GFP aggregates}

The relative aggregation scores of protein-GFP were quantified at the single-cell level (based on the cell segmentation) using the same methodology as for Yap1 and Msn2 nuclear scores. The relative quantification of GFP foci upon stress exposures had been previously assessed following a similar method in S. Saad et al., Nat Cell Biol, 2017. We checked that the dynamics was not depending on the number of pixels accounting for the GFP foci. The averaged signal represents the mean of all cells present in the different field of view (multi-position acquisition).

\section{Quantification of the SRX1pr-GFP-deg signal and redox adaptation index quantification}

The SRX1pr-GFP-deg signal was assessed at the single-cell level (based on the segmentation). The GFP signal of each cell was obtained by quantifying the mean fluorescence of the pixels included in the segmented cell area.

The redox adaptation index (RAI) was quantified at the single-cell level. For each cell, the mean GFP value after $5 \mathrm{~h}$ under $0.1 \mathrm{mM} \mathrm{H} 2 \mathrm{O} 2$ was divided by the mean GFP value after $1 \mathrm{~h}$ under 0.1 $\mathrm{mM} \mathrm{H} 2 \mathrm{O} 2$. The score was then quantified relative to the WT and plotted as RAI (delta strain) / RAI (WT). A small H2O2 adaptation index represents a weak ability to rescue redox equilibrium. The dose of $\mathrm{H} 2 \mathrm{O} 2$ was chosen as low as $0.1 \mathrm{mM}$ so that every strain included could grow under stress, testifying of their adaptation. We checked that the SRX1pr-GFP was not expressed under $\mathrm{H} 2 \mathrm{O} 2$ in a yap1 delta strain (data not shown), testifying of the specificity of the transcriptional response toward the Yap1 regulon and $\mathrm{H} 2 \mathrm{O} 2$ sensing.

\section{Acknowledgments}

We thank Michel Toledano for fruitful discussions, Sophie Quintin and Sandrine Morlot for careful reading of the manuscript. This work was supported by the Fondation pour la Recherche Médicale (FRM, B.J, and G.C.), the Agence Nationale pour la Recherche (T.A. and G.C.), the grant ANR-10-LABX-0030-INRT, a French State fund managed by the Agence Nationale de la Recherche under the frame program Investissements d'Avenir ANR-10-IDEX0002-02.

\section{References}

Balaban, Nathalie Q., Sophie Helaine, Kim Lewis, Martin Ackermann, Bree Aldridge, Dan I. Andersson, Mark P. Brynildsen, et al. 2019. "Definitions and Guidelines for Research on Antibiotic Persistence." Nature Reviews. Microbiology 17 (7): 441-48.

Balaban, Nathalie Q., Jack Merrin, Remy Chait, Lukasz Kowalik, and Stanislas Leibler. 2004. "Bacterial Persistence as a Phenotypic Switch." Science 305 (5690): 1622-25.

Bigger, Josephw. 1944. "TREATMENT OF STAPHYLOCOCCAL INFECTIONS WITH PENICILLIN BY INTERMITTENT STERILISATION.” The Lancet 244 (6320): 497-500.

Bodvard, Kristofer, Ken Peeters, Friederike Roger, Natalie Romanov, Aeid Igbaria, Niek Welkenhuysen, Gael Palais, et al. 2017. "Light-Sensing via Hydrogen Peroxide and a 
Peroxiredoxin." Nature Communications 8 (March): 14791.

Boisnard, Stéphanie, Gilles Lagniel, Cecilia Garmendia-Torres, Mikael Molin, Emmanuelle BoyMarcotte, Michel Jacquet, Michel B. Toledano, Jean Labarre, and Stéphane Chédin. 2009. "H2O2 Activates the Nuclear Localization of Msn2 and Maf1 through Thioredoxins in Saccharomyces Cerevisiae." Eukaryotic Cell 8 (9): 1429-38.

Brauner, Asher, Ofer Fridman, Orit Gefen, and Nathalie Q. Balaban. 2016. "Distinguishing between Resistance, Tolerance and Persistence to Antibiotic Treatment." Nature Reviews. Microbiology 14 (5): 320-30.

Broach, James R. 2012. "Nutritional Control of Growth and Development in Yeast." Genetics 192 (1): 73-105.

Chae, H. Z., S. J. Chung, and S. G. Rhee. 1994. "Thioredoxin-Dependent Peroxide Reductase from Yeast." The Journal of Biological Chemistry 269 (44): 27670-78.

Conrad, Michaela, Joep Schothorst, Harish Nag Kankipati, Griet Van Zeebroeck, Marta RubioTexeira, and Johan M. Thevelein. 2014. "Nutrient Sensing and Signaling in the Yeast Saccharomyces Cerevisiae." FEMS Microbiology Reviews 38 (2): 254-99.

Decker, Carolyn J., and Roy Parker. 2012. "P-Bodies and Stress Granules: Possible Roles in the Control of Translation and mRNA Degradation." Cold Spring Harbor Perspectives in Biology 4 (9): a012286.

Delaunay, A., A. D. Isnard, and M. B. Toledano. 2000. "H2O2 Sensing through Oxidation of the Yap1 Transcription Factor." Science's STKE: Signal Transduction Knowledge Environment 19 (19): 5157.

Erjavec, Nika, Lisa Larsson, Julie Grantham, and Thomas Nyström. 2007. “Accelerated Aging and Failure to Segregate Damaged Proteins in Sir2 Mutants Can Be Suppressed by Overproducing the Protein Aggregation-Remodeling Factor Hsp104p." Genes \& Development 21 (19): 2410-21.

Garcia-Barrio, M., J. Dong, S. Ufano, and A. G. Hinnebusch. 2000. "Association of GCN1GCN20 Regulatory Complex with the N-Terminus of elF2alpha Kinase GCN2 Is Required for GCN2 Activation." The EMBO Journal 19 (8): 1887-99.

Gasch, A. P., P. T. Spellman, C. M. Kao, O. Carmel-Harel, M. B. Eisen, G. Storz, D. Botstein, and P. O. Brown. 2000. "Genomic Expression Programs in the Response of Yeast Cells to Environmental Changes." Science's STKE: Signal Transduction Knowledge Environment 11 (12): 4241.

Godon, C., G. Lagniel, J. Lee, J. M. Buhler, S. Kieffer, M. Perrot, H. Boucherie, M. B. Toledano, and J. Labarre. 1998. "The H2O2 Stimulon in Saccharomyces Cerevisiae." The Journal of Biological Chemistry 273 (35): 22480-89.

Goulev, Youlian, Sandrine Morlot, Audrey Matifas, Bo Huang, Mikael Molin, Michel B. Toledano, and Gilles Charvin. 2017. "Nonlinear Feedback Drives Homeostatic Plasticity in $\mathrm{H}_{2} \mathrm{O} 2$ Stress Response." eLife 6 (April). https://doi.org/10.7554/eLife.23971.

Gray, Joseph V., Gregory A. Petsko, Gerald C. Johnston, Dagmar Ringe, Richard A. Singer, and Margaret Werner-Washburne. 2004. "Sleeping Beauty': Quiescence in Saccharomyces Cerevisiae." Microbiology and Molecular Biology Reviews: MMBR 68 (2): 187-206.

Guan, Qiaoning, Suraiya Haroon, Diego González Bravo, Jessica L. Will, and Audrey P. Gasch. 2012. "Cellular Memory of Acquired Stress Resistance in Saccharomyces Cerevisiae." Genetics 192 (2): 495-505.

Hall, Andrea, P. A. Karplus, and Leslie B. Poole. 2009. "Typical 2-Cys Peroxiredoxins-Structures, Mechanisms and Functions." The FEBS Journal 276 (9): 2469-77.

Hanzén, Sarah, Katarina Vielfort, Junsheng Yang, Friederike Roger, Veronica Andersson, Sara Zamarbide-Forés, Rebecca Andersson, et al. 2016. "Lifespan Control by Redox-Dependent Recruitment of Chaperones to Misfolded Proteins." Cell 166 (1): 140-51.

Hasan, Rukhsana, Christophe Leroy, Anne-Dominique Isnard, Jean Labarre, Emmanuelle Boy- 
Marcotte, and Michel B. Toledano. 2002. "The Control of the Yeast H2O2 Response by the Msn2/4 Transcription Factors." Molecular Microbiology 45 (1): 233-41.

Hohmann, Stefan. 2002. "Osmotic Stress Signaling and Osmoadaptation in Yeasts." Microbiology and Molecular Biology Reviews: MMBR 66 (2): 300-372.

Iraqui, Ismail, Guy Kienda, Jérémie Soeur, Gérard Faye, Giuseppe Baldacci, Richard D. Kolodner, and Meng-Er Huang. 2009. "Peroxiredoxin Tsa1 Is the Key Peroxidase Suppressing Genome Instability and Protecting against Cell Death in Saccharomyces Cerevisiae." PLoS Genetics 5 (6): e1000524.

Jacquet, Michel, Georges Renault, Sylvie Lallet, Jan De Mey, and Albert Goldbeter. 2003. "Oscillatory Nucleocytoplasmic Shuttling of the General Stress Response Transcriptional Activators Msn2 and Msn4 in Saccharomyces Cerevisiae." The Journal of Cell Biology 161 (3): 497-505.

Jang, Ho Hee, Kyun Oh Lee, Yong Hun Chi, Bae Gyo Jung, Soo Kwon Park, Jin Ho Park, Jung Ro Lee, et al. 2004. "Two Enzymes in One; Two Yeast Peroxiredoxins Display Oxidative Stress-Dependent Switching from a Peroxidase to a Molecular Chaperone Function." Cell 117 (5): 625-35.

Jiang, Heng, and Ann M. English. 2006. "Phenotypic Analysis of the ccp1 $\Delta$ and $\operatorname{ccp} 1 \Delta$ ccp1W191F Mutant Strains of Saccharomyces Cerevisiae Indicates That Cytochrome c Peroxidase Functions in Oxidative-Stress Signaling." Journal of Inorganic Biochemistry 100 (12): 1996-2008.

Kuehne, Andreas, Hila Emmert, Joern Soehle, Marc Winnefeld, Frank Fischer, Horst Wenck, Stefan Gallinat, et al. 2015. "Acute Activation of Oxidative Pentose Phosphate Pathway as First-Line Response to Oxidative Stress in Human Skin Cells." Molecular Cell 59 (3): 35971.

Kuge, S., and N. Jones. 1994. "YAP1 Dependent Activation of TRX2 Is Essential for the Response of Saccharomyces Cerevisiae to Oxidative Stress by Hydroperoxides." The EMBO Journal 13 (3): 655-64.

Lee, J., C. Godon, G. Lagniel, D. Spector, J. Garin, J. Labarre, and M. B. Toledano. 1999. "Yap1 and Skn7 Control Two Specialized Oxidative Stress Response Regulons in Yeast." The Journal of Biological Chemistry 274 (23): 16040-46.

Levy, Sasha F., Naomi Ziv, and Mark L. Siegal. 2012. "Bet Hedging in Yeast by Heterogeneous, Age-Correlated Expression of a Stress Protectant." PLoS Biology 10 (5): e1001325.

Milias-Argeitis, Andreas, Sean Summers, Jacob Stewart-Ornstein, Ignacio Zuleta, David Pincus, Hana El-Samad, Mustafa Khammash, and John Lygeros. 2011. "In Silico Feedback for in Vivo Regulation of a Gene Expression Circuit." Nature Biotechnology 29 (12): 1114-16.

Minard, K. I., G. T. Jennings, T. M. Loftus, D. Xuan, and L. McAlister-Henn. 1998. "Sources of NADPH and Expression of Mammalian NADP+-Specific Isocitrate Dehydrogenases in Saccharomyces Cerevisiae." The Journal of Biological Chemistry 273 (47): 31486-93.

Molin, Mikael, Junsheng Yang, Sarah Hanzén, Michel B. Toledano, Jean Labarre, and Thomas Nyström. 2011. "Life Span Extension and $\mathrm{H}(2) \mathrm{O}(2)$ Resistance Elicited by Caloric Restriction Require the Peroxiredoxin Tsa1 in Saccharomyces Cerevisiae." Molecular Cell 43 (5): 823-33.

Muzzey, Dale, Carlos A. Gómez-Uribe, Jerome T. Mettetal, and Alexander van Oudenaarden. 2009. "A Systems-Level Analysis of Perfect Adaptation in Yeast Osmoregulation." Cell 138 (1): 160-71.

Nissan, Tracy, Purusharth Rajyaguru, Meipei She, Haiwei Song, and Roy Parker. 2010. "Decapping Activators in Saccharomyces Cerevisiae Act by Multiple Mechanisms." Molecular Cell 39 (5): 773-83.

Pedrajas, J. R., A. Miranda-Vizuete, N. Javanmardy, J. A. Gustafsson, and G. Spyrou. 2000. "Mitochondria of Saccharomyces Cerevisiae Contain One-Conserved Cysteine Type Peroxiredoxin with Thioredoxin Peroxidase Activity." The Journal of Biological Chemistry 
275 (21): 16296-301.

Ralser, Markus, Mirjam M. Wamelink, Axel Kowald, Birgit Gerisch, Gino Heeren, Eduard A. Struys, Edda Klipp, et al. 2007. "Dynamic Rerouting of the Carbohydrate Flux Is Key to Counteracting Oxidative Stress." Journal of Biology 6 (4): 10.

Roger, Friederike, Cecilia Picazo, Wolfgang Reiter, Marouane Libiad, Chikako Asami, Sarah Hanzén, Chunxia Gao, et al. 2020. "Peroxiredoxin Promotes Longevity and H2O2Resistance in Yeast through Redox-Modulation of Protein Kinase A." eLife 9 (July). https://doi.org/10.7554/eLife.60346.

Slatkin, Montgomery. 1974. "Hedging One's Evolutionary Bets." Nature 250 (5469): 704-5.

Tamaki, Hisanori. 2007. "Glucose-Stimulated cAMP-Protein Kinase A Pathway in Yeast Saccharomyces Cerevisiae." Journal of Bioscience and Bioengineering 104 (4): 245-50.

Toledano, Michel B., Agnès Delaunay, Ludivine Monceau, and Frédérique Tacnet. 2004. "Microbial H2O2 Sensors as Archetypical Redox Signaling Modules." Trends in Biochemical Sciences 29 (7): 351-57.

Toledano, Michel B., Anne-Gaëlle Planson, and Agnès Delaunay-Moisan. 2010. "Reining in $\mathrm{H}(2) \mathrm{O}(2)$ for Safe Signaling." Cell 140 (4): 454-56.

Veal, Elizabeth A., Alison M. Day, and Brian A. Morgan. 2007. "Hydrogen Peroxide Sensing and Signaling." Molecular Cell 26 (1): 1-14.

Wong, Chi-Ming, Yuan Zhou, Raymond W. M. Ng, Hsiang-Fu Kung Hf, and Dong-Yan Jin. 2002. "Cooperation of Yeast Peroxiredoxins Tsa1p and Tsa2p in the Cellular Defense against Oxidative and Nitrosative Stress." The Journal of Biological Chemistry 277 (7): 5385-94.

\section{Figure legends}

\section{Figure 1: Resistance and tolerance are distinct properties of the response to hydrogen peroxide}

(A) Schematic representation of the difference between oxidative stress tolerance (ability of cells to survive stress without proliferation) and stress resistance (ability of cells to proliferate and adapt under the stressor). (B) and (C) Fold change in cell number under $\mathrm{H}_{2} \mathrm{O}_{2}$. In $\mathrm{B}$, stress continues until the end of the experiment. In $\mathrm{C}$, stress is released after $1 \mathrm{~h}$. Time is normalized from stress beginning. Stripes indicate the stress pattern and the $\mathrm{H}_{2} \mathrm{O}_{2}$ concentration. Error bars represent the SEM of $\mathrm{N}=3$ independent technical replicates. (D) Time course of single yeast cell's behaviour under $\mathrm{H}_{2} \mathrm{O}_{2}$ and after stress release (duration of $\mathrm{H}_{2} \mathrm{O}_{2}$ bolus $=4 \mathrm{~h}$ ). The singlecell approach enables to distinguish Fold change proliferation (in red) from post-stress survival 
(in blue). The ability of a cell (born before that the stress begins) to form a colony after stress release accounts for one surviving event. (E) Survival fraction of WT cells for different stress duration (from 0 to $8 \mathrm{~h}$ ) and different concentrations $(0.5 \mathrm{mM}$ and $1 \mathrm{mM}$, below and above the MIC(WT), respectively). Log scale.

\section{Figure 2: A stress pattern modulation framework to reveal the determinants of oxidative stress resistance and oxidative stress tolerance}

(A) Schematic of the stress-pattern modulation methodology used. Cells were exposed to two transient boluses of $0.5 \mathrm{mM} \mathrm{H}_{2} \mathrm{O}_{2}$, increasing the stress concentration sharply (step assay) or slowly (ramp assay). (B) Normalized cell proliferation under stress and post-stress survival were quantified as two independent readouts of oxidative stress resistance and oxidative stress tolerance, respectively (see also Methods). Quantification of the post-stress survival in both step and ramp assays (see also Methods). $N=12$ independent replicates, $n>100$ for each replicate (C) Scheme of screen used to decipher the stress response genes involved in both stress tolerance and resistance. (D) Quantification of the post-stress survival in the mutants screened both in the step and the ramp assays. Log scale. (E) Quantification of the normalized cell proliferation in mutant strains screened both in the step and the ramp assays. D and E; the dashed line represents the condition with a similar response in both step and ramp assays, the yellow circle represents adapter strains, exhibiting a response at least 1.5 times better in the ramp assay, the purple circle represents non-adapter strains, not filling the criterion sub-cited. (F) Time series of strains carrying PrSRX1-GFP-deg, imaged in phase contrast, and GFP fluorescence. Curves represent the corresponding quantification of the median GFP signals within single cells (black lines) and the median response within the population. The single-cells traces are only representative and do not include all trajectories included in the median value. ( $n>50$ cells for each condition). The $\mathrm{H}_{2} \mathrm{O}_{2}$ adaptation index (HAI) is calculated as the mean GFP value after $1 \mathrm{~h}$ under stress divided by the mean GFP value after $5 \mathrm{~h}$ under stress $\left(\mathrm{H}_{2} \mathrm{O}_{2} 0.1\right.$ $\mathrm{mM}$ ). (G) Quantification of the proliferative capacity of cells in the ramp assay as a function of the $\mathrm{H}_{2} \mathrm{O}_{2}$ adaptation index, both parameters relative to the WT score. The $\mathrm{R}^{2}$ value represents the square of the correlation between the response values and the predicted response values with a linear fitting. $(\mathbf{H})$ Quantification of the post-stress survival in response to the step assay as a function of the $\mathrm{H}_{2} \mathrm{O}_{2}$ adaptation index, both parameters relative to the WT score. The $\mathrm{R}^{2}$ value represents the square of the correlation between the response values and the predicted response values with a linear fitting. $B, D, E, G$ and $H$; error bars represent the SEM. D, E, G and $\mathrm{H}$; At least 3 independent technical replicates.

\section{Figure 2 Suppl:}

(A) Typical image sequences of a BY4742 Wild Type strain observed by time-lapse microscopy in a microfluidic device (phase contrast). The strain is observed in the step and the ramp assays. Growth is greatly impacted in the step assay compared to the ramp assay. The white bar represents $6.2 \mu \mathrm{m}$. Curves represent the fold cell number under step and ramp assays. Error bar represents the SEM on independent technical replicates. (B) Scheme of the experimental protocol used to assess oxidative stress resistance and tolerance. (C) Examples of different mutant strains (WT, pde $2 \Delta$, tsa1 $\Delta$ and zwf $1 \Delta$ ) screened under oxidative stress following the framework described in A. Proliferation under stress and post-stress survival was 
assessed. The white bar represents $6.2 \mu \mathrm{m}$. (D) Quantification of the post-stress survival of cells in response to the ramp assays as a function of the redox adaptation index, both parameters relative to WT score. The $\mathrm{r}^{2}$ value represents the square of the correlation between the response values and the predicted response values with a linear fitting. (E) Survival fraction kinetics of both a WT and a zwf1 strain in log scale as a function of the stress duration. MDK99 represents the minimal duration of stress to kill $99 \%$ of the population (see Methods). The dashed line is an exponential fit, the continuous line a bi-exponential fit. (F) Screen of the MDK99 for WT and zwf1 $\Delta$ strains in response to different doses of $\mathrm{H} 2 \mathrm{O} 2$, from 0.25 to $128 \mathrm{mM}$. At least 2 independent technical replicates were averaged for each time point.

\section{Figure 3: The high tolerance of the zwf1 $\Delta$ mutant is accompanied by a reduced oxidative stress resistance and a redox imbalance under low and mild doses of $\mathrm{H} 2 \mathrm{O} 2$}

(A) Time series of WT and zwf $1 \Delta$ strains under $0.1 \mathrm{mM} \mathrm{H}_{2} \mathrm{O}_{2}$, more than five hours following stress beginning. Phase contrast and SRX1pr-GFP signals are shown as two separate channels. Two newly-formed buds are marked with orange arrows. (B) Single-cell quantification of the bud to bud frequency (see Methods) under the indicated dose of stress after cells reached equilibrium, in both WT and $z w f 1 \Delta$ strains. Circles represent means $+/-$ the standard deviation. The dash lines represent the smallest frequency considered as a cell-cycle event (a bud-to-bud frequency of $0.0017 \mathrm{~min}^{-1}$, corresponding to a $10 \mathrm{~h}$ division time). (C) Quantification of the median SRX1pr-GFP-deg fluorescence expression inside single yeast cells, 6h after being submitted to the indicated dose of $\mathrm{H}_{2} \mathrm{O}_{2}$. The circles (blue and red) represent the mean $+/-$ the standard deviation. (D) Bud to bud frequency in single cells as a function of the maximal fluorescence expression of the SRX1pr-GFP-deg reporter during the corresponding cell cycle. Log scale. Open circles correspond to cells evolving without $\mathrm{H}_{2} \mathrm{O}_{2}$ (WT in blue, zwf1 $\Delta$ in red), filled circles correspond to cells evolving under $0.1 \mathrm{mM} \mathrm{H}_{2} \mathrm{O}_{2}$ (WT in blue, zwf1 $\Delta$ in red). For the in-stress condition, cell-cycle events were only included after $6 \mathrm{~h}$ under stress to ensure cells had reached equilibrium. (E) and (F) Time series of Tsa1-GFP in WT and $z w f 1 \Delta$ strains and Hsp104-GFP respectively, under $0.5 \mathrm{mM} \mathrm{H}_{2} \mathrm{O}_{2}$. Stress duration is indicated with the stripe. The time is normalized to stress the beginning. The white bar represents $6.2 \mu \mathrm{m}$. The curves correspond to the quantifications of the aggregation score of both protein-GFP reporters under stress (see Methods). Mean GFP signals in the population +/- SEM are represented by the bold lines and the small lines respectively. (G) Time series of a zwf $1 \Delta$ recovering growth after stress release (after $16 \mathrm{~h}$ under stress), with or without adding DTT $(5 \mathrm{mM})$ to the glucose $2 \%$ medium. The Hsp104-GFP aggregate is represented with orange arrows. The first bud observed after stress release is marked with an orange arrow. The time between stress release and cell-cycle reentry is indicated in orange. The white bar represents $6.2 \mu \mathrm{m}$. (H) The corresponding Hsp104 aggregation scores after stress release, with or without DTT $5 \mathrm{mM}$. Only cells that adapted were included in the analysis. The Hsp104 aggregation scores were normalized by the value measured at the moment of stress release in every single cell. The bold and small red (without DTT) and purple (with DTT) lines represent respectively the mean value and the SEM of the Hsp104 aggregation scores within the two post-stress surviving populations. The dash black line (without DTT) and the continuous black line (with DTT) represent the cumulative fraction of cells that re-entered in the cell-cycle (see Methods). (I) Left panel: Histogram of the basal (before stress) Srx1-GFP signal in WT and $z w f 1 \Delta$ strains $(\mathrm{N}=535)$. Right panel: $z w f 1 \Delta$ single-cell 
signals (from the left panel) were normalized by the average fluorescence value within the whole $z w f 1 \Delta$ population and used as a proxy of the redox basal imbalance to predict post stress survival (see scheme). Single cells were binned into 5 categories as a function of their prestress GFP expression and survival score were plotted in each binned population. $\mathrm{N}=107$ cells for each bin. The vertical error bar represents the standard deviation of the survival fraction, approximated by a bootstrap procedure. The horizontal error bar represents the SEM of the fluorescence signal within each binned population. The $R^{2}$ value represents the square of the correlation between the response values and the predicted response values with a linear fitting.

\section{Figure 3 Suppl}

(A) Determination of the resistance limit of a $z w f 1 \Delta$ strain. The fold cell number is quantified under various $\mathrm{H}_{2} \mathrm{O}_{2}$ stress concentrations to assess the $\mathrm{MIC}$ (from 0 to $0.4 \mathrm{mM}$, MIC $0.3 \mathrm{mM}$ ). The response of a WT strain at $0.4 \mathrm{mM}$ is also represented. (B) Determination of the resistance limit of a trr1 $\Delta$ strain. The fold cell number is quantified under various $\mathrm{H}_{2} \mathrm{O}_{2}$ stress concentrations to assess the MIC (from 0 to $0.4 \mathrm{mM}, \mathrm{MIC}<0.1 \mathrm{mM}$ ). The response of a WT strain at $0.4 \mathrm{mM}$ is also represented. (C) Yap1sf-GFP nuclear localization score (see Methods) in WT and zwf $1 \Delta$ strains under 0.2 or $0.5 \mathrm{mM} \mathrm{H}_{2} \mathrm{O}_{2}$. Stripes indicate the stress period.

\section{Figure 4}

(A) On the left, images of Msn2-GFP merged with the phase-contrast signal, before or under 0.5 mM H2O2 (30 min after stress beginning) in a WT strain and a pde2 $\Delta$ strain +/- cAMP $3 \mathrm{mM}$. The white bar represents $6.2 \mu \mathrm{m}$. Time is normalized from stress beginning. On the right, the corresponding quantification of the nuclear score of Msn2-GFP over time. The stripe indicates the stress pattern. The time is normalized from stress beginning. Mean GFP nuclear localization score in the population +/- SEM are represented by the bold lines and the small lines respectively. (B) Same than (A) with WT and zwf $1 \Delta$, zwf $1 \Delta$ pde2 $\Delta+/-$ cAMP $3 \mathrm{mM}$ mutant strains (C) Mean bud to bud frequency under stress (+/- standard deviation) as a function of the mean SRX1pr-GFP expression (+/- standard deviation) under different $\mathrm{H} 2 \mathrm{O} 2$ stress conditions (from 0 to $0.3 \mathrm{mM}$ ) for a WT strain and $z$ wf $1 \Delta$, zwf1 $1 \Delta$ pde2 $\Delta$ mutant strains. The dash lines represent the smallest frequency considered as a cell-cycle event (a bud-to-bud frequency of $0.0017 \mathrm{~min}^{-1}$, corresponding to a 10h division time). (D) Quantification of the SRX1pr-GFP-deg signals within single-cells under $0.3 \mathrm{mM} \mathrm{H} 2 \mathrm{O} 2$. (E) and (F) Post-stress survival fraction in a step assay $(E)$ and in a ramp assay $(F)$. Open circles represent independent technical replicates, and the bar represents the mean value of the replicates $(n>100$ for each replicate). Error bar represents the SEM. Stripes represent stress patterns. (G) Survival fractions in both step and ramp assays in the indicated mutants. $N>3$ (at least) independent technical replicates for each condition. Stripes represent stress patterns. (H) Scheme of the PKA pathway downstream elements screened for tolerance in both a WT and a zwf1 $\Delta$ contexts. Red squares represent the downstream elements required for the increased oxidative stress tolerance observed in a $z w f 1 \Delta$ strain. (I) Post stress survival fractions in response to a $2 \mathrm{~h} \mathrm{H}_{2} \mathrm{O}_{2}$ stress bolus $(1 \mathrm{mM})$. Filled circles represent technical replicates $(N=2$ or $3, n>100$ per replicate), error bar represents the SEM of replicates. (J) Quantification of the Pat1-GFP aggregation score within the corresponding populations, relative to the WT score (see Methods). The line indicates the median within the population. The statistical analysis used a one-side Man Whitney $U$ test to challenge whether each mutant had a greater aggregation or not than the WT strain. Only the 
zwf1 $\Delta$ score was significantly greater than the WT score $\left(p<0.0001,{ }^{* \star *}\right)$. Other $p$-values were $>$ 0.05 (NS). (K) The corresponding images of Pat1-GFP before and under $1 \mathrm{mM} \mathrm{H}_{2} \mathrm{O}_{2}$ stress (1h after stress beginning) in a WT and the different mutant strains. Dash white circle indicates cellular contours.

\section{Figure 4 Suppl}

(A) Boxplots of the fold enrichment in the Msn2-GFP nuclear score between pre-stress condition and 30min after stress beginning ( $n>50$ for each condition). (B) Quantification of the SRX1pr-GFP-deg signals within single-cells under $0.3 \mathrm{mM} \mathrm{H} 2 \mathrm{O} 2$. (C) Post-stress survival quantification. The stress pattern is indicated with the scheme (7.5 min, 32 or $64 \mathrm{mM})$. Filled circles represent independent technical replicates, the error bars represent SEMs. (D) Boxplots of the fold enrichment in the Msn2-GFP nuclear score between pre-stress condition and 30min after stress beginning ( $n>50$ for each condition). (E) Post-stress survival quantification in the indicated mutant strains. The stress pattern is indicated with the stripe. 


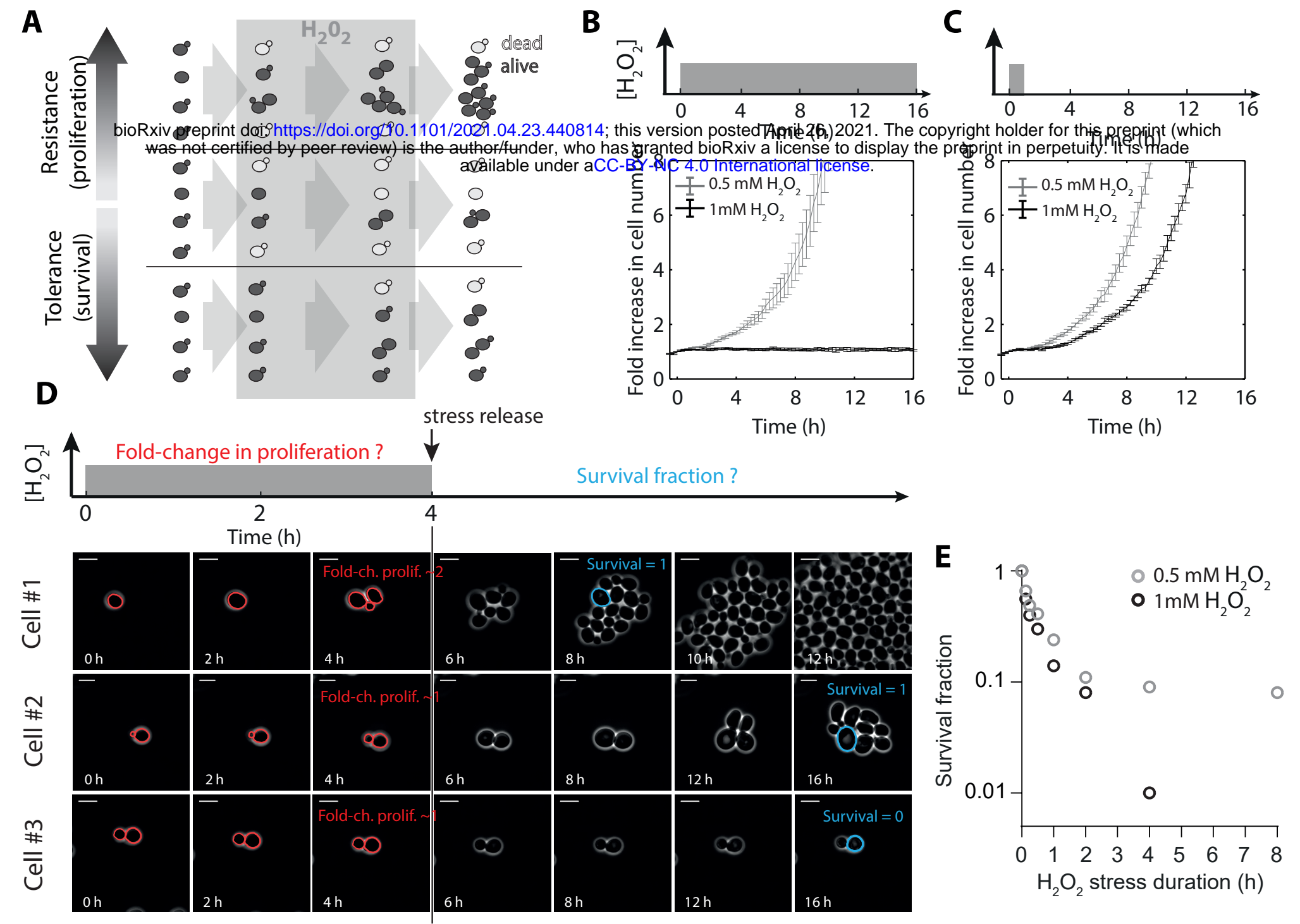


A

ত $\sim$ Step

bioRxiv preprint doi: was not certifiedfypeer review) is thea author/funder, who has granted bioRxiv a (cense to display the preprint in perpetuity. It is made available under $\mathrm{aCC}-\mathrm{B}^{\mathrm{K}}-\mathrm{NC} 4.0$ International license.

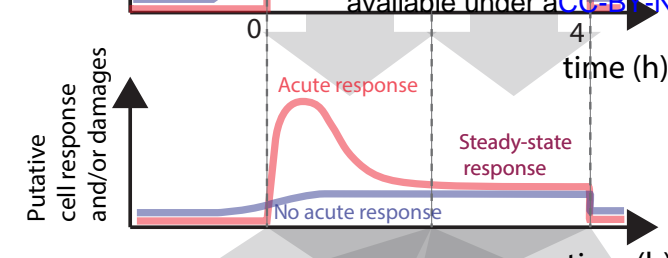

B
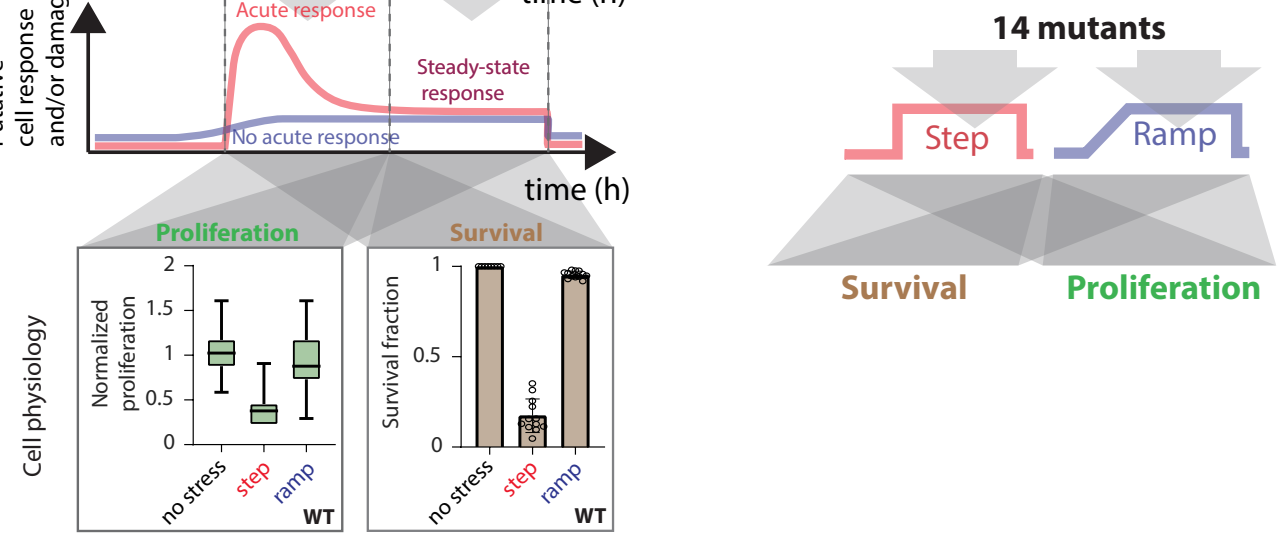

D

Survival

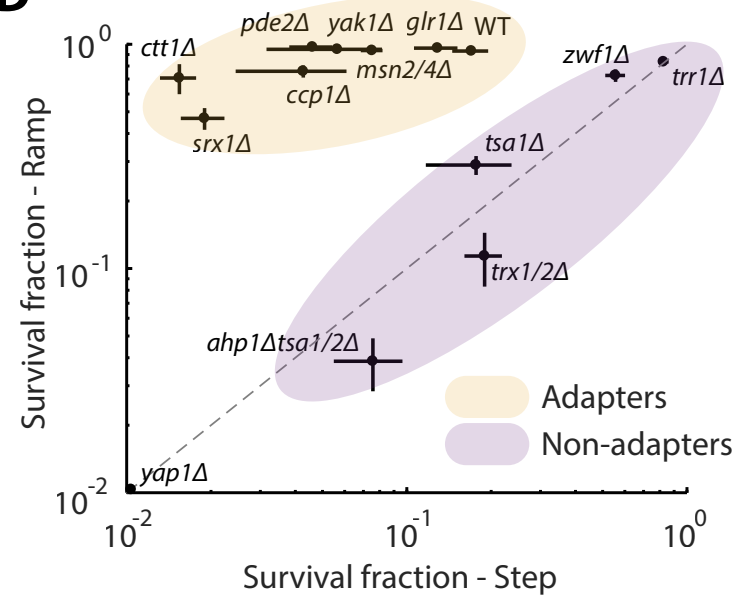

E

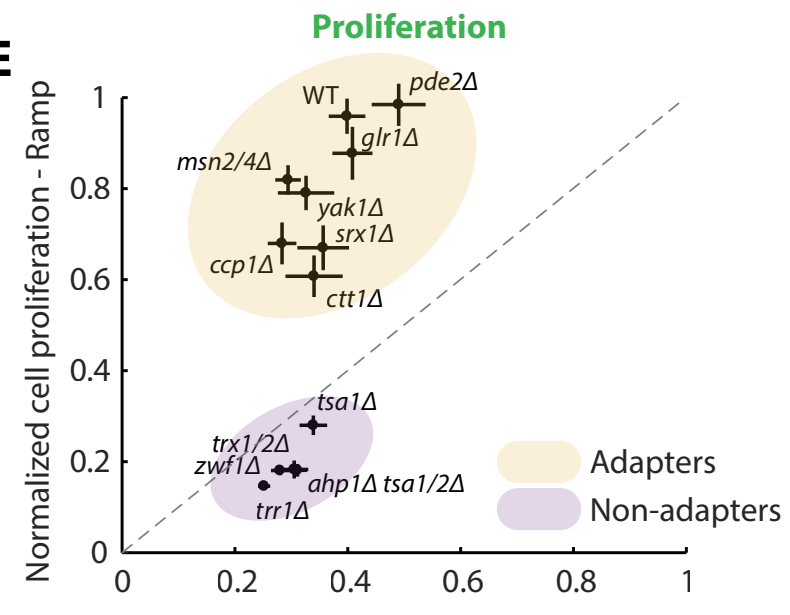

$\mathbf{F}$

Survival fraction - Step

Normalized cell proliferation - Step
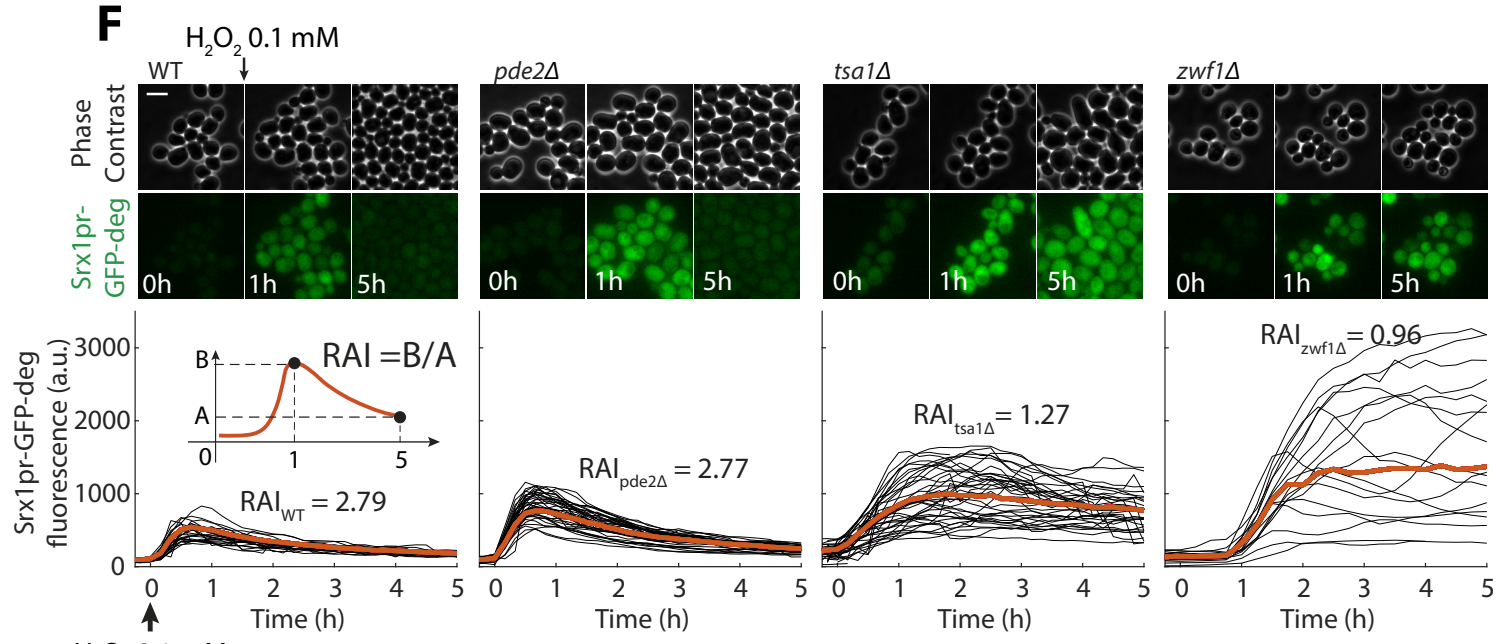
$\mathrm{H}_{2} \mathrm{O}_{2} 0.1 \mathrm{mM}$

G

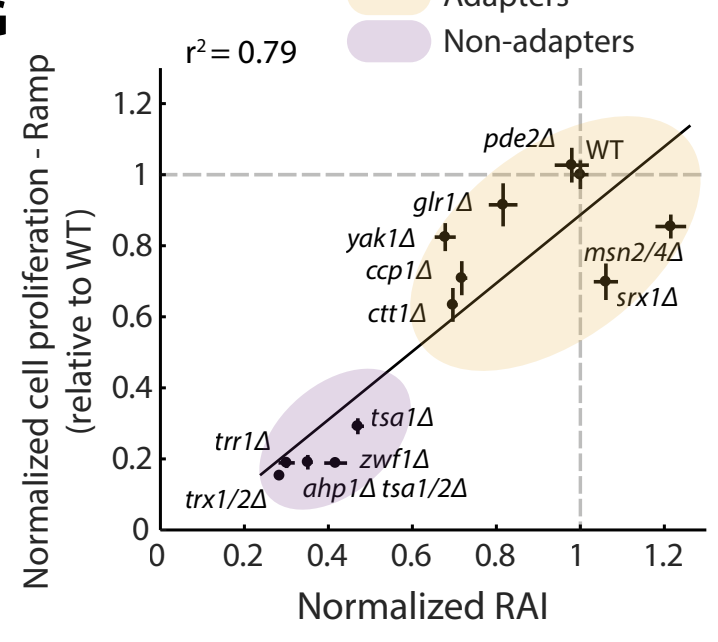

Adapters

H

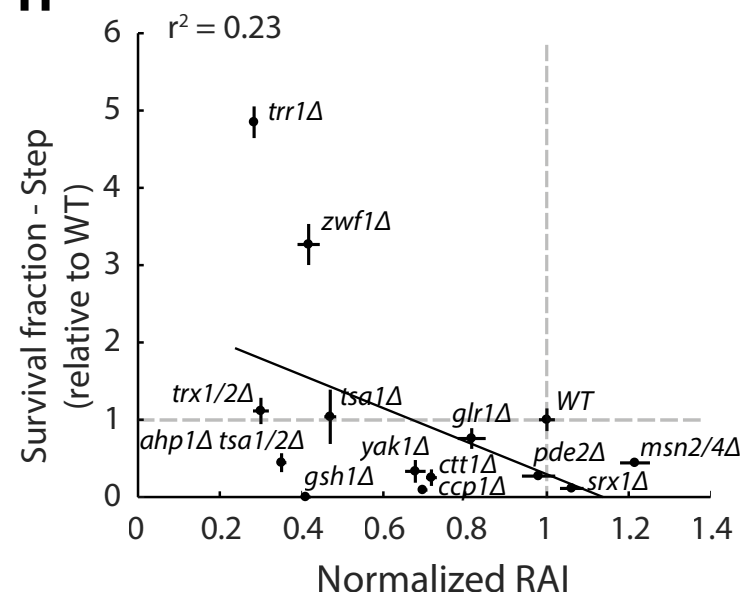


$\begin{array}{lll}\text { A Phase Contrast } & \text { B } \\ \text { Srx1 pr-GFP-deg }\end{array}$

Srx1pr-GFP-deg Cell cycle duration $=120 \mathrm{~min}$

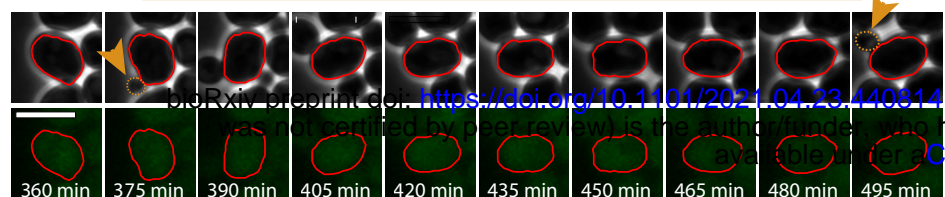

Cell cycle duration $=315 \mathrm{~min}$

WT cell, $0.1 \mathrm{mM}$

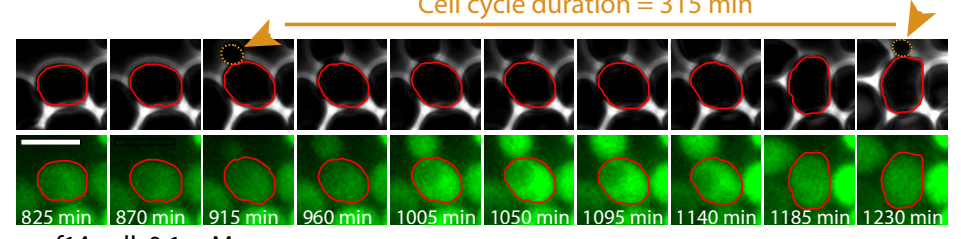

zwf1 $1 \Delta$ cell, $0.1 \mathrm{mM}$

C

$-W T-z w f 1 \Delta$

- WT - zwf1

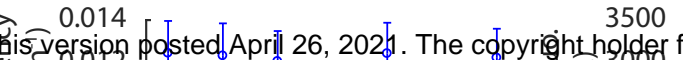

this yersion possted April 26, 202 . The coppyrighth holder for this preprint (which as grantedibiokxiv a license to dlsplay the prepripit $3 n$ perpetuity. It is made Cक्ष N0.910 International license.

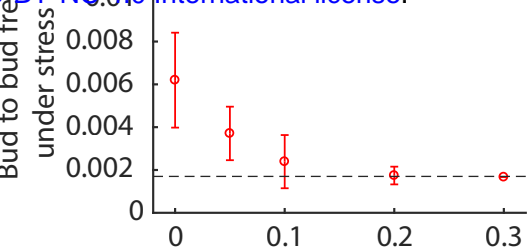

$\mathrm{H}_{2} \mathrm{O}_{2}$ concentration (mM)

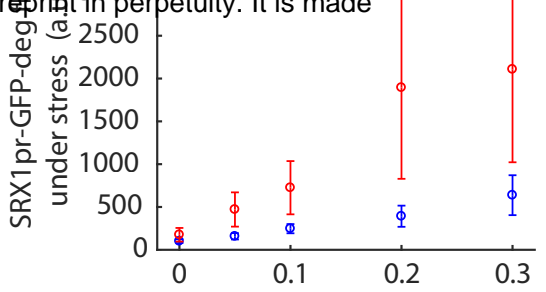

$\mathrm{H}_{2} \mathrm{O}_{2}$ concentration (mM)
D

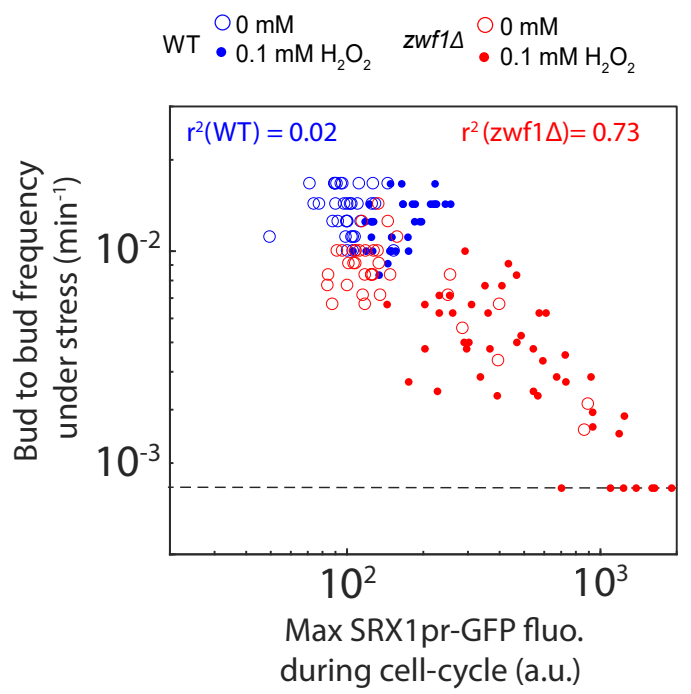

E

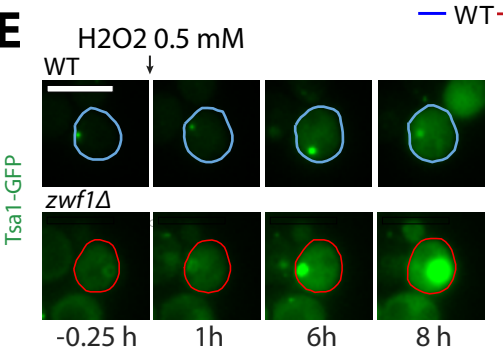

$\mathbf{F}$

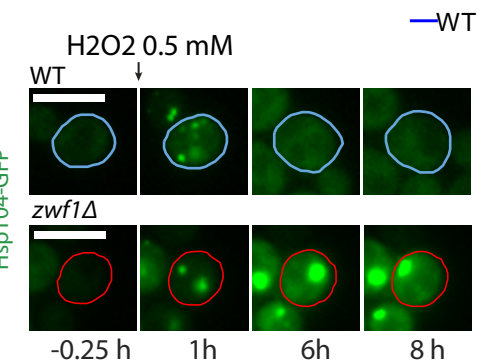

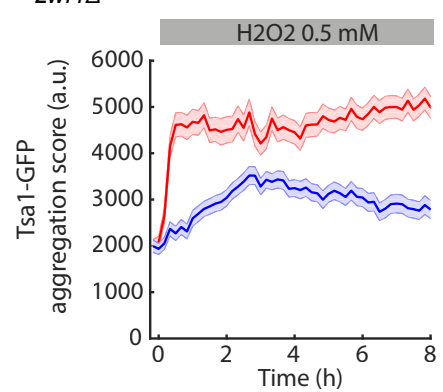

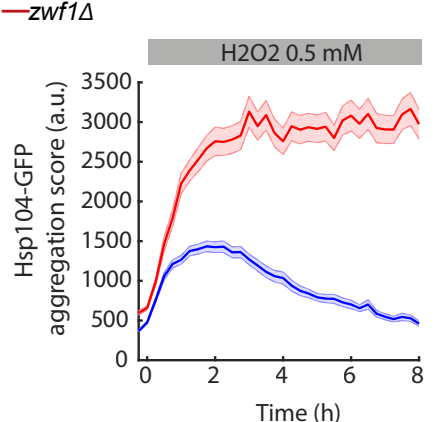

G stress release

$\downarrow$ zwf1 $1,2 \%$ glucose after stress release

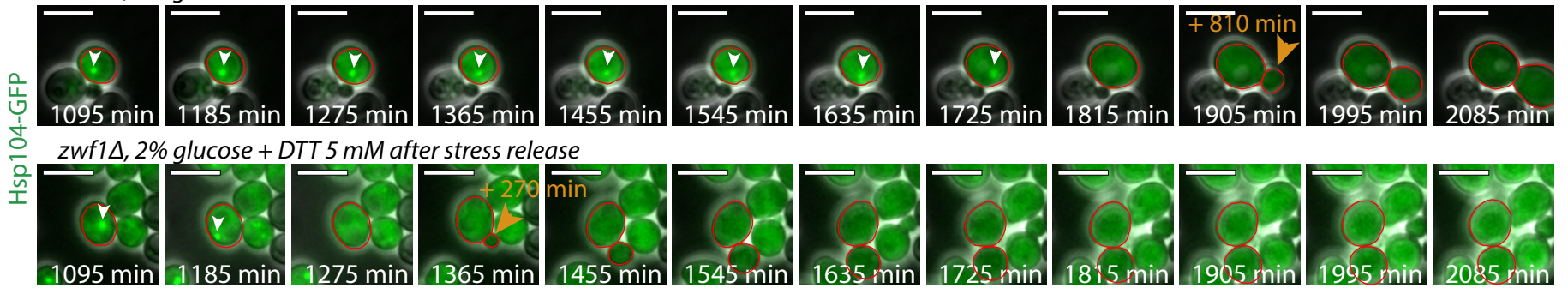

- glucose $2 \%$

- - - glucose $2 \%$

— glucose $2 \%+$ DTT

— glucose $2 \%$ + DTT
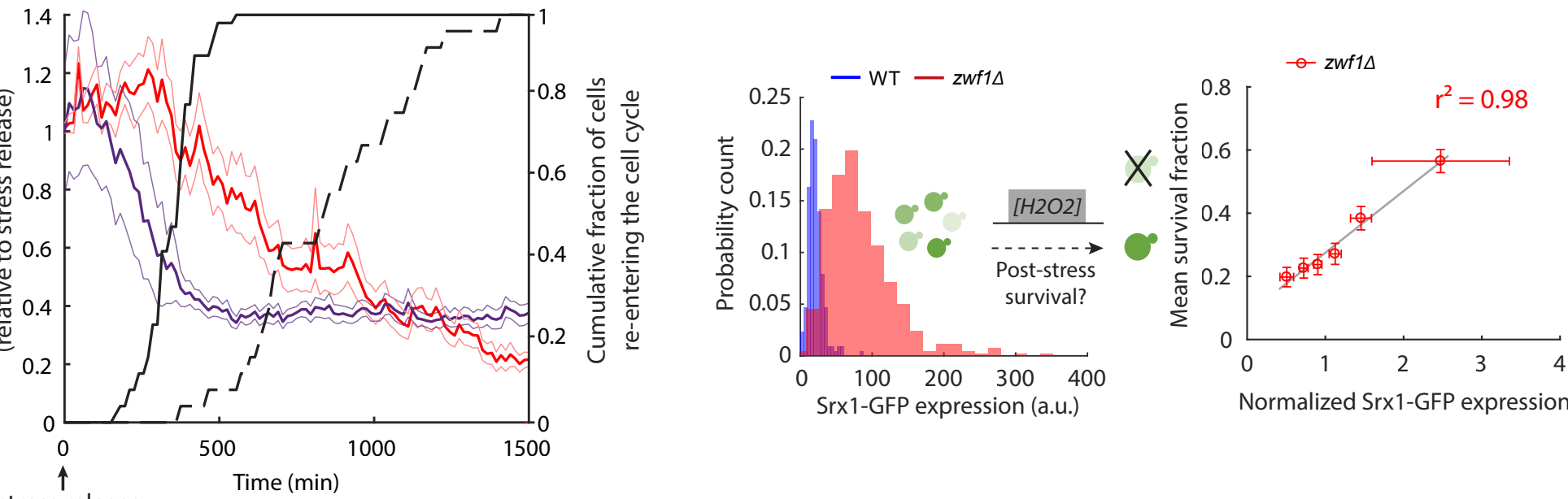

Normalized Srx1-GFP expression

stress release 
A Msn2-GFP

+ Phase Contrast

- WT

- pde2 $\Delta$

- pde $2 \Delta+$ cAMP 3 mM

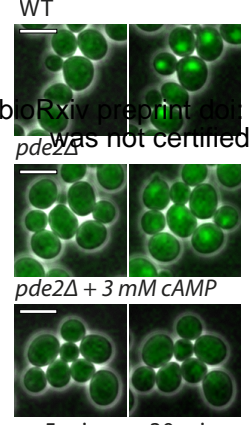

$-5 \mathrm{~min} \quad 30 \mathrm{~min}$

\section{$600 \quad \mathrm{H} 2 \mathrm{O} 20.5 \mathrm{mM}$}

https://60. drg/10.1101/2021.04.23.440814; this version

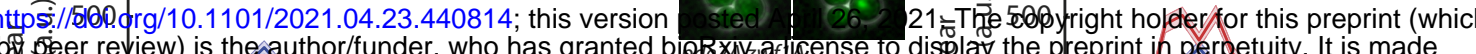

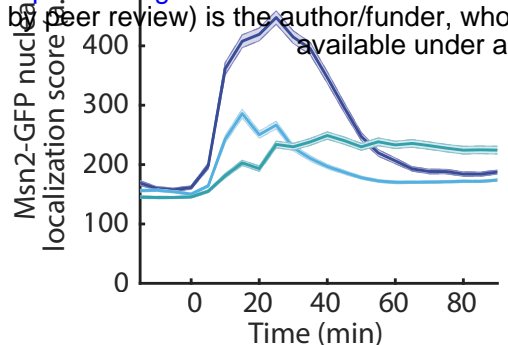

C
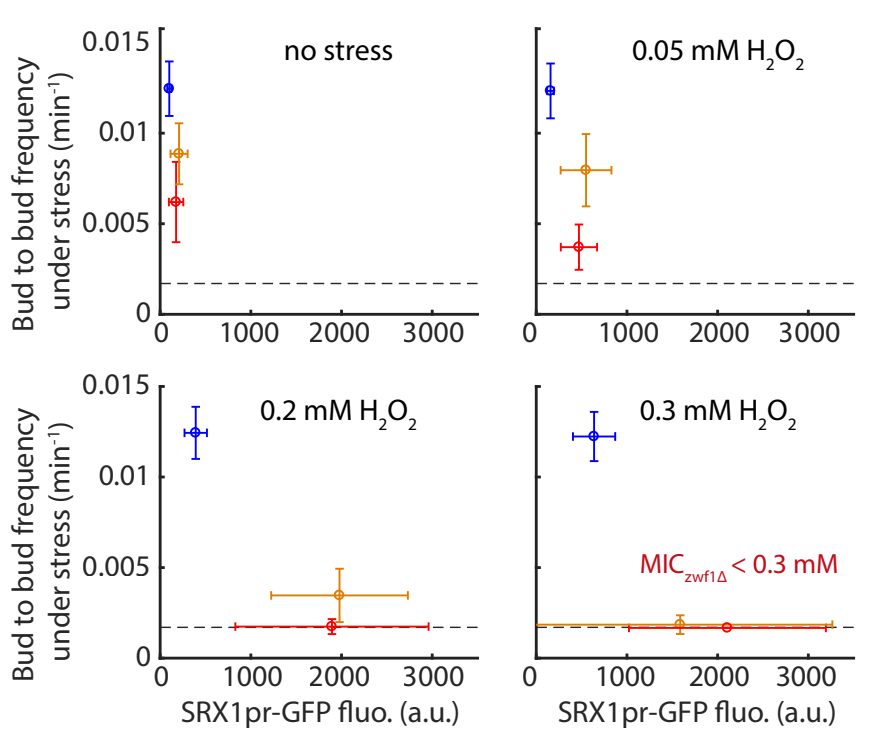

$\mathbf{E}$
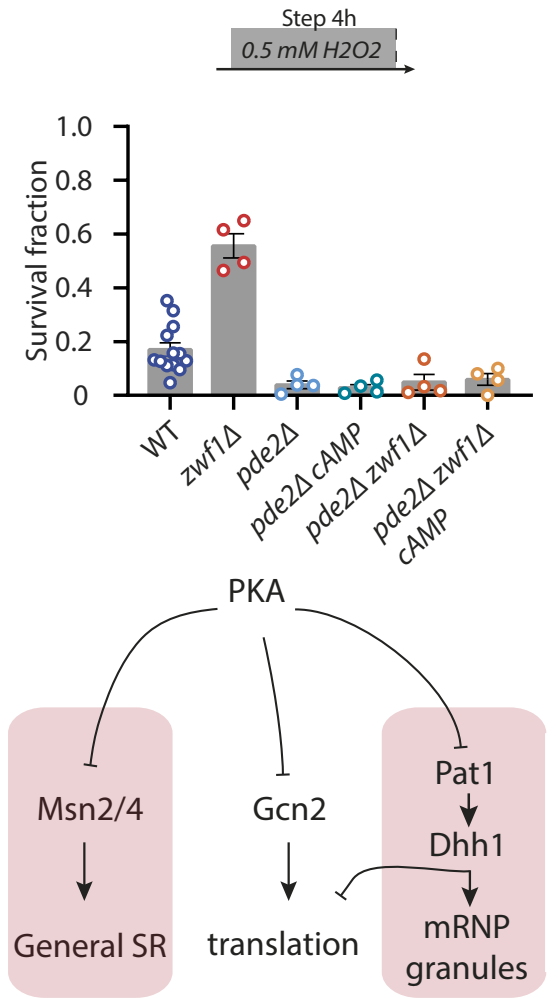

B Msn2-GFP

+ Phase Contrast

zwfi $1 \Delta$
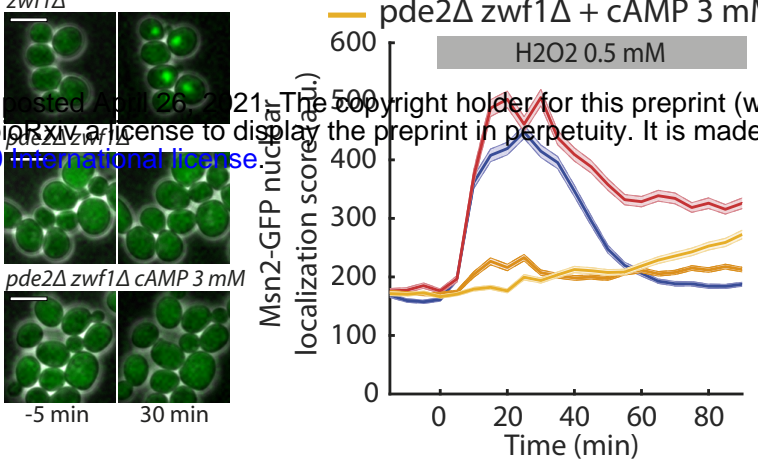

- WT

- pde $2 \Delta$ zwf1 $\Delta$

- pde2 $\Delta$ zwf1 $\Delta+$ cAMP $3 \mathrm{mM}$

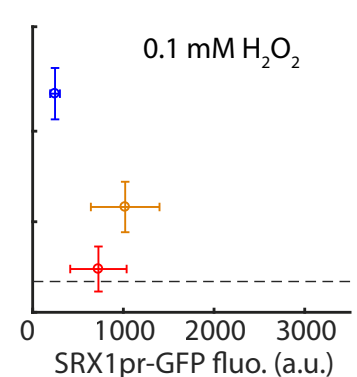

D $0.3 \mathrm{mM} \mathrm{H}_{2} \mathrm{O}_{2}$
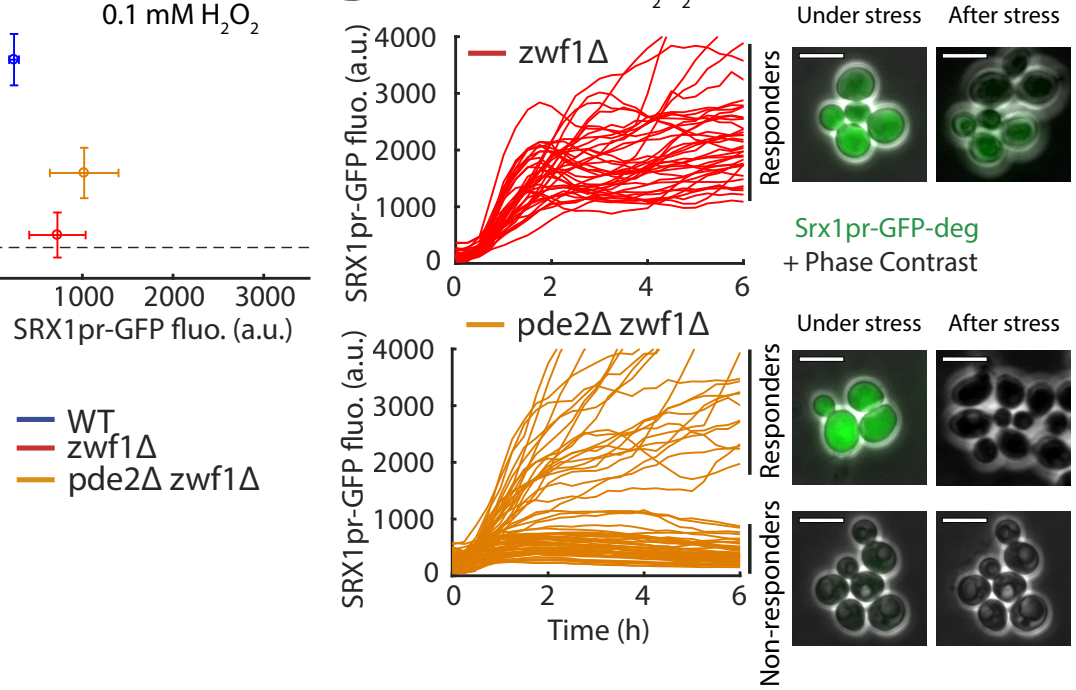

- pde2 2 zwf1 $\Delta$

SRX1 pr-GFP fluo. (a.u.)
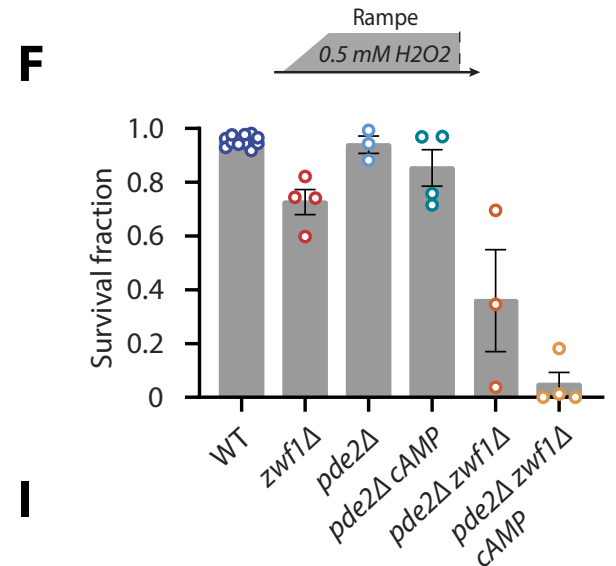

G
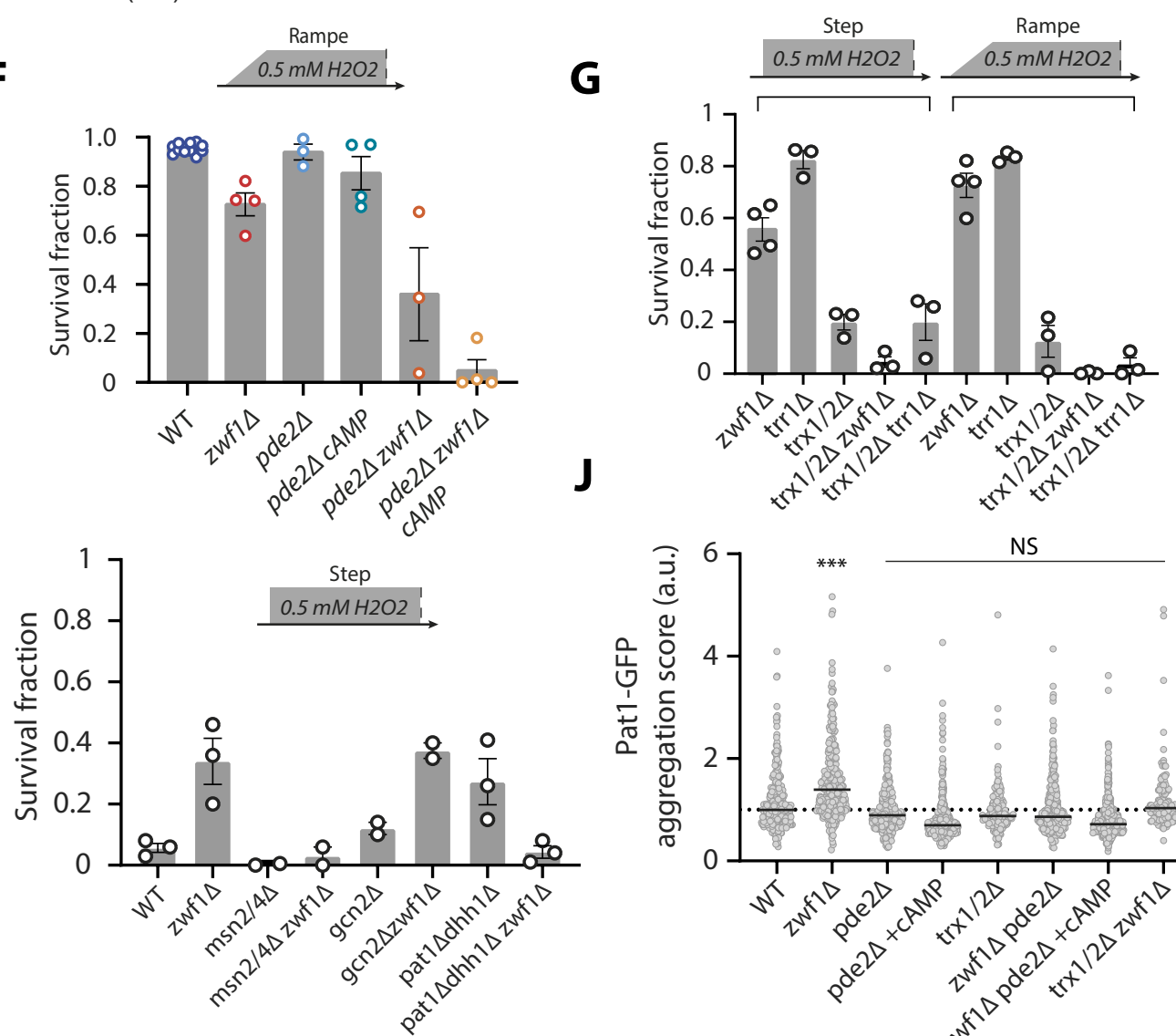

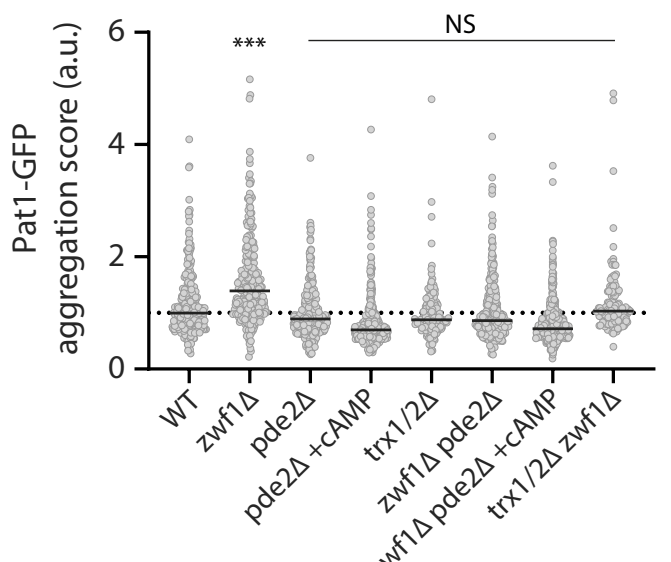

zwf1 $\triangle$ pde2 $\triangle$
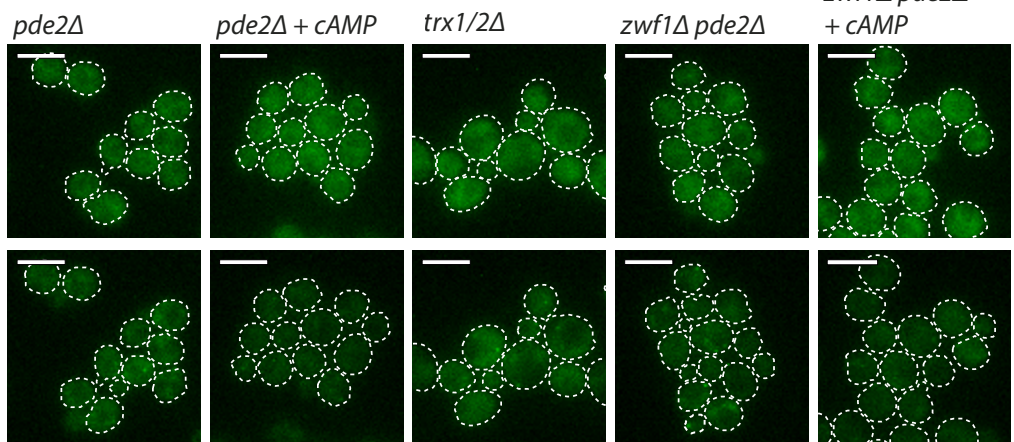

$z w f 1 \Delta \operatorname{trx} 1 / 2 \Delta$
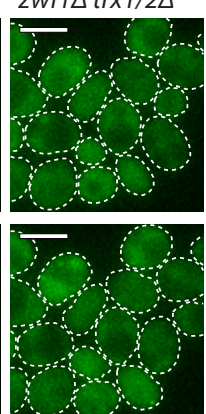
A

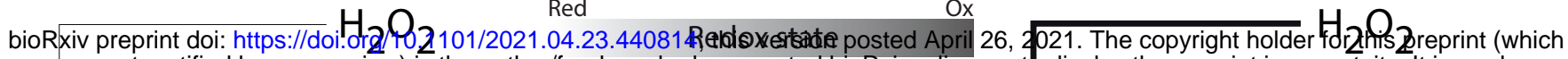
was not certified by peer review is the author/funder, who has granted bioRxiv alicense to display the preprint in perpetuity. It is made

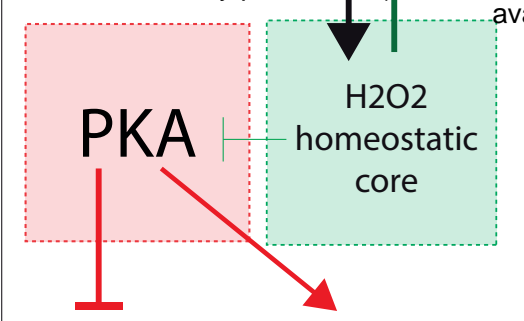
Survival Proliferation
(tolerance) (resistance)
Trxred
$\mathrm{NADPH}$
$\mathrm{NADP}^{+}$
$\mathrm{H} 2 \mathrm{O} 2$ hom. core function
H2O2 scavenging Redox signaling
Antagonistic
states

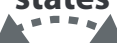
PKA activity

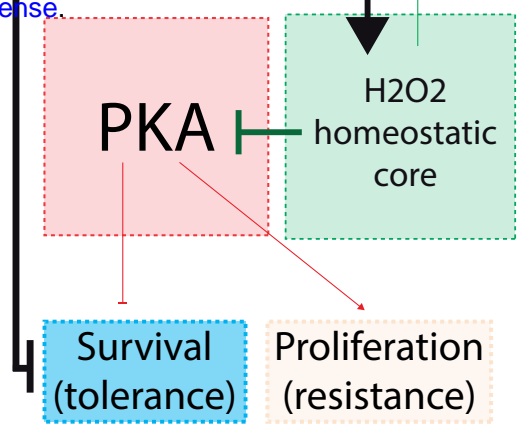

